\title{
No arousal-biased competition in focused visuospatial attention
}

\author{
Árni Gunnar Ásgeirsson ${ }^{1,2}$ \& Sander Nieuwenhuis ${ }^{1}$
}

1: Cognitive Psychology Unit, Leiden University, The Netherlands

2: University of Akureyri, Iceland.

Corresponding author:

Árni Gunnar Ásgeirsson

arnigunnar@unak.is

University of Akureyri

Sólborg v/ Nordurslod

IS-600

Akureyri

Iceland

Sander Nieuwenhuis

Cognitive Psychology Unit

Faculty of Social Sciences

Leiden University

Word Count: 12652 
Arousal sometimes enhances and sometimes impairs perception and memory. A recent theory attempts to reconcile these findings by proposing that arousal amplifies the competition between stimulus representations, strengthening already strong representations and weakening already weak representations. Here, we report a stringent test of this arousal-biased competition theory in the context of focused visuospatial attention. Participants were required to identify a briefly presented target in the context of multiple distractors, which varied in the degree to which they competed for representation with the target, as revealed by psychophysics. We manipulated arousal using emotionally arousing pictures (Experiment 1), alerting tones (Experiment 2) and white-noise stimulation (Experiment 3), and validated these manipulations with electroencephalography and pupillometry. In none of the experiments did we find evidence that arousal modulated the effect of distractor competition on the accuracy of target identification. Bayesian statistics revealed moderate to strong evidence against arousal-biased competition. Modeling of the psychophysical data based on Bundesen's (1990) theory of visual attention corroborated the conclusion that arousal does not bias competition in focused visuospatial attention.

Keywords: alerting, emotion, phasic arousal, tonic arousal, cognitive modeling, Bayesian statistics. 


\section{Introduction}

Arousal, the global state of activation of our central and autonomic nervous system, is one of the driving forces in human behavior. Recent years have seen a renewed interest in the effects of arousal on brain, mind and behavior (Cavanagh, Wiecki, Kochar, \& Frank, 2014; Eldar, Cohen, \& Niv, 2013; Lee, Baek, Lu, \& Mather, 2014; Murphy, Vandekerckhove, \& Nieuwenhuis, 2014; Nassar, Rumsey, Wilson, Parikh, Heasly, \& Gold, 2012; Sørensen, Vangkilde, \& Bundesen, 2015; Vinck, Batista-Brito, Knoblich, \& Cardin, 2015; Warren et al., 2016). These studies have revealed that slow as well as second-to-second fluctuations in arousal have highly specific influences on neural activity and cognitive function. A common theme in this research is that arousal level modulates the impact of new observations on subsequent perceptual inferences, a finding that has led to detailed computational models in which arousal indexes specific forms of uncertainty and corresponding changes in gain or estimated precision (e.g., Nassar et al., 2012; Allen et al., 2016; Murphy, Boonstra, \& Nieuwenhuis, 2016). A less well understood aspect of arousal is that it sometimes enhances and sometimes impairs perception and memory (reviewed in Hanoch \& Vitouch, 2004; Mather \& Sutherland, 2011; Mather, Clewett, Sakaki, \& Harley, 2016). For example, the same arousal manipulation may enhance perceptual learning of a target among dissimilar distractors, while impairing perceptual learning of the same target among similar distractors (Lee, Itti, \& Mather, 2012). Here, we examine this aspect of arousal by investigating how arousal shapes visual perception of targets and distractors in a focused-attention task. 
An elegant theory that attempts to explain the somewhat contradictory effects of arousal on cognitive function is the arousal-biased competition theory (henceforth $\mathrm{ABC}$ theory; Mather \& Sutherland, 2011). ABC theory is based on the idea of biased competition (e.g., Bundesen, 1990; Desimone, \& Duncan, 1995) which views visual attention as a competitive process, during which a processing capacity of a fixed size is divided asymmetrically among signals of varying interest (or priority). Because processing capacity is fixed, a processing advantage of one signal must come at the expense of processing other signals. Building on classic arousal studies (Bacon, 1974; Easterbrook, 1959; Hockey \& Hamilton, 1970), ABC theory posits that the competitive advantages caused by biased competition are further exaggerated under arousal, leading to "winner-take-more/loser-take-less" dynamics (Mather \& Sutherland, 2011). The priority of a given signal relative to other signals is what determines whether it will be amplified or attenuated by arousal. When a signal is assigned high priority, either due to its salience (e.g, intensity) or goal relevance (Fecteau \& Munoz, 2006), then arousal will amplify this signal, leading to a competitive advantage in biased competition.

Conversely, when the salience or relevance of the signal is low, arousal will attenuate it, further impeding behavioral responses to that signal.

$\mathrm{ABC}$ theory has several virtues. First, the key principle of arousal-biased competition is consistent with a class of computational models in which the modulatory effects of catecholamine-mediated changes in arousal are implemented as a change in the responsivity or gain of task-processing units, and as a result produce the winner-takemore/loser-take-less effects that ABC theory attempts to explain (Eldar et al., 2103; 4 
Servan-Schreiber, Printz, \& Cohen, 1990; Warren, Murphy, \& Nieuwenhuis, 2016).

Second, Mather and colleagues have proposed a biologically plausible account of how the winner-take-more/loser-take-less effects of arousal-biased competition are realized in the brain (Mather et al., 2016). A third major advantage of $A B C$ theory is that it attempts to explain the effects of arousal on a wide range of cognitive processes, including perception, attention, and memory, and may even apply to higher-order cognition such as strategy use in decision-making (Wichary, Mata, \& Rieskamp, 2015).

To date, the clearest evidence for the ABC theory is seen in memory research. For example, Sakaki, Fryer, and Mather (2014; see also Clewett, Sakaki, Nielsen, Petzinger, \& Mather, 2017) presented a serial stream of pictures of which one was an oddball (signified by a black frame), and asked half of the participants to prioritize the oddball itself, and the other half to prioritize the picture preceding it (oddball-1). The researchers found that the arousal induced by the oddball picture affected memory for the oddball-1 picture in a way that depended on whether subjects prioritized that item. The arousing picture facilitated memory of the preceding neutral picture in the group of participants that prioritized those oddball-1 pictures, while it impaired memory of the oddball-1 picture in the group that prioritized the oddballs rather than the preceding neutral items. This data pattern can be accounted for in terms of arousal-biased competition by positing that arousal enhanced memory consolidation of the prioritized signal representations at the expense of other, lower-priority signal representations.

In this article we focus on the potential of $\mathrm{ABC}$ theory to account for effects of arousal on another cognitive process: visual attention. Sutherland and Mather (2012) 5 
conducted a direct test of arousal-biased competition principles in visual attention. They presented participants with unpleasant and neutral sounds before flashing eight target letters on the screen. Of the eight targets, three were high-contrast letters, while the other five were of low contrast. This manipulation was assumed to force asymmetric bottom-up prioritization of letters, in that the strong, high-contrast signals would naturally be prioritized above the weaker, low-contrast signals. Participants were instructed to report as many of the letters as possible, but were not asked to value one letter type over the other. The authors found that high-contrast letters were more likely to be reported, but also that this bias was amplified following arousing sounds. The opposite was true for low-contrast letters, which were reported less often under arousal. Another dividedattention study found that increases in temporal attention, which are accompanied by increased arousal, enhance the efficiency of selecting targets rather than distractors (Sørensen et al., 2015). This pattern of findings provides some promising first evidence that arousal increases competition in divided visual attention, amplifying the effects of prioritization based on (bottom-up) salience or (top-down) task-relevance.

In the current study, we assessed whether the principles of arousal-biased competition also apply in the context of focused rather than divided visuospatial attention. Specifically, our participants were forced to exert a high degree of attentional control to select a target from irrelevant distractors of differing intensity. We present the results of three main experiments (Experiments 1A, 2 and 3A) and two control experiments (Experiments 1B and 3B) using this focused-attention task in which we employed three different, well-established arousal manipulations to examine whether 6 
arousal modulates the competition for perceptual representation of stimuli differing in both top-down (task relevance) and bottom-up (salience) priority.

The specific task that we used was a singleton letter identification task, in which we briefly presented a red target letter, either 1) alone (target alone condition; Figure 1.3), 2) flanked by five blue distractor letters (homogeneous distractor condition; Figure 1.4), or 3) flanked by four blue and a single yellow distractor letter (salient distractor condition; Figure 1.5).

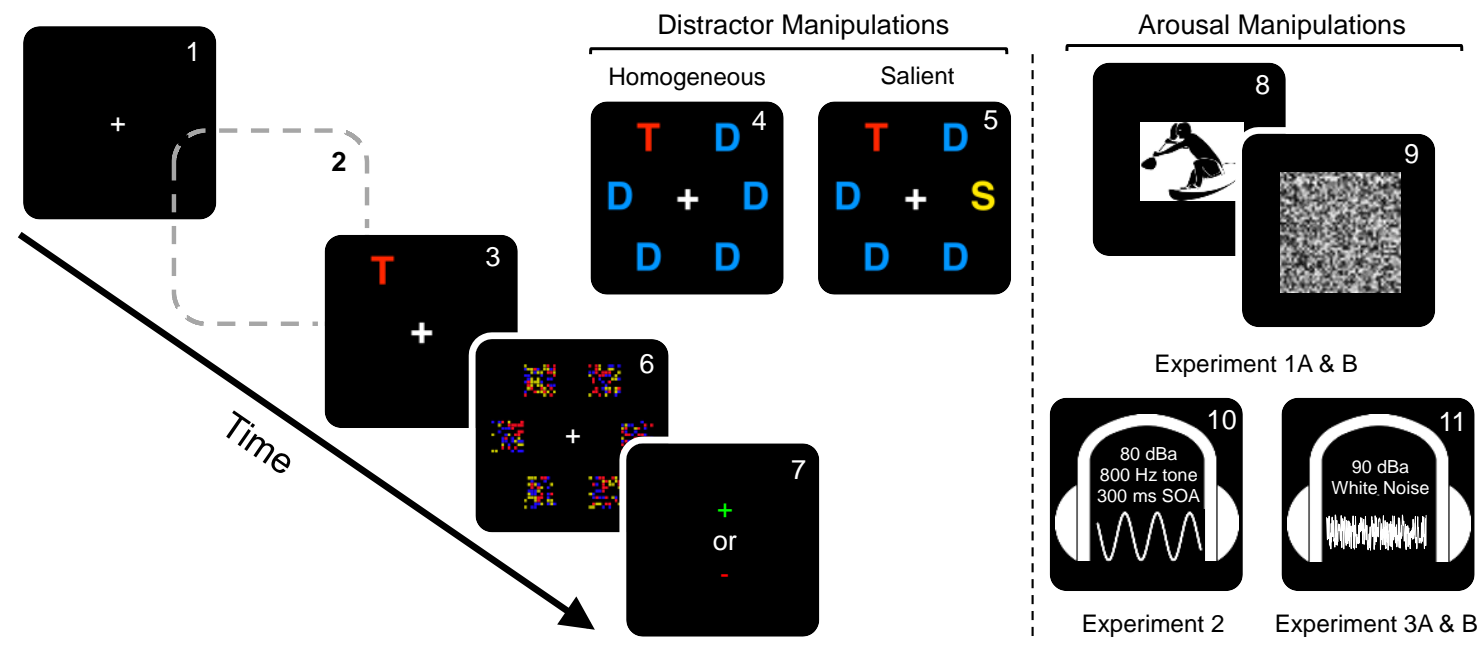

Fig. 1. The general progression of a trial in all three experiments. The left panel shows stages of the procedure of all experiments. Stages 3-5 show the different conditions of distraction: the target alone condition (3), the homogeneous distractor condition (4), and the salient distractor condition (5). Note that the $T$ 's, $D$ 's and $S$ are symbolic of targets, distractors and salient distractor. In the experiment, the identities of letter stimuli were randomly chosen from all letters of the English alphabet. The right panel shows the arousal manipulations that were unique to each experiment. In Experiments 1A, 1B (arousing pictures) and 2 (an alerting tone), the arousing - or control - stimuli were inserted at stage 2. In Experiments 3A and 3B, white noise was present/absent throughout whole blocks of the experimental task. Stimuli are not drawn to scale nor in their true colors (see Stimuli and Apparatus for details).

By manipulating levels of distraction we were able to gauge perceptual performance when there was no, medium, and hard competition for limited attentional resources. 
In Experiments 1A and 1B arousal was manipulated by presentation of pleasant, unpleasant and neutral pictures from the International Affective Picture System (IAPS; Lang, Bradley, \& Cuthbert, 2008). The IAPS picture set has been used successfully to induce emotional arousal in a large number of studies on visual cognition (e.g., Kristjánsson, Óladóttir, \& Most, 2012; Lee, et al., 2012). Compared to the International Affective Digital Sounds (IADS) stimulus set used by Sutherland and Mather (2012), the IAPS is a much larger set of stimuli and supports category formations (e.g., pleasant, unpleasant and neutral) with a sharper distinction between arousal and valence ratings. Therefore, we reasoned that - if anything - arousal effects should be increased by using IAPS pictures, relative to IADS sounds.

In Experiment 2 we used auditory alerting tones to induce arousal in participants, and compared task performance to a no-tone condition. A loud tone often induces a reflexive phasic arousal response (Hackley \& Valle-Inclán, 2003; Tona, Murphy, Brown, \& Nieuwenhuis, 2016), and can serve as a temporal cue for the participant to concentrate their efforts in a narrow interval of time.

Finally, in Experiments 3A and 3B we examined the effects of a tonic arousal manipulation on focused visuospatial attention. We presented ongoing loud white noise to participants while they performed the singleton letter identification task, and compared performance to blocks without auditory stimulation. Although most of the support for $\mathrm{ABC}$ theory is based on phasic arousal manipulations, Mather and Sutherland (2011; p. 120) mention an experiment by Hockey (1970), who found that ongoing loud auditory noise facilitated responses to higher-probability centrally presented stimuli while 8 
impairing responses to lower-probability peripherally presented stimuli. This effect of loud noise on selectivity was not found when central and peripheral stimuli were presented with equal likelihood. Although Hockey manipulated expectations rather than bottom-up or top-down attention, his result has been interpreted as a narrowing of attention under arousal (noise), in line with Easterbrook's (1959) cue-utilization theory of arousal (see Kahneman, 1973; p. 37-42, for a short review). Note that according to ABC theory, the result reflects an arousal-induced attentional bias toward high-priority (here: high-probability) stimuli, not necessarily a narrowing of attentional focus.

In all three experiments we tested the prediction, derived from $\mathrm{ABC}$ theory, that arousal would modulate the effects of competition (level of distraction) on task performance. The prediction of an interaction between arousal and level of distraction was statistically evaluated using repeated-measures ANOVAs as well as their Bayesian counterparts (Rouder, Morey, Verhagen, Swagman, \& Wagenmakers, in press), from which we obtained quantitative evidence for each of three models that might plausibly explain the data (see General Method-Bayesian Analysis). Furthermore, to get a richer sense of the cognitive processes underlying performance in the experiments, we formally modeled the data based on the theory of visual attention (TVA), which enabled us to examine arousal-related modulations of the cognitive mechanisms involved in selective attention (Bundesen, 1990). The TVA model has been successful at accounting for various aspects of visual attention, including feature selectivity (Shibuya \& Bundesen, 1988), temporal expectation (Sørensen et al., 2015; Vangkilde, Petersen, \& Bundesen, 2013), attention in brain-lesioned patients (Bublak et al., 2005; Duncan et al., 1999), 9 
inter-trial priming (Ásgeirsson, Kristjánsson, \& Bundesen, 2014, 2015), salient singleton processing (Nordfang, Dyrholm, \& Bundesen, 2013), for performance in monitoring for visual events of different salience (Poth, Petersen, Bundesen, \& Schneider, 2014), agerelated decline (Wiegand et al., 2014), and dyslexia (Bogon, Finke, \& Stenneken, 2014; see Bundesen \& Habekost, 2014 and Habekost, 2015, for recent general and clinically focused reviews of the literature on TVA, respectively).

\section{General Method}

\subsection{Participants}

Participation was limited to 18-30 year olds. All participants reported normal or corrected-to-normal vision, and normal color vision. Participants were excluded if they had acute or chronic neural disorders or used psychoactive drugs. They were asked not to consume caffeinated beverages in the hour leading up to the experimental participation. Compensation was provided in the form of a cash payment in proportion to the duration of the experimental session, or by course credit. All participants were informed of their rights in accordance with the Declaration of Helsinki. The study was approved by the 
Ethics Committee of the Institute of Psychology at Leiden University (CEP number 9501177439).

\subsection{Stimuli and Apparatus}

The experimental tasks were written and executed in MATLAB, using the Psychophysics Toolbox (Brainard, 1997; Kleiner et al., 2007; Pelli, 1997). The behavioral experiments, Experiments 1A, 2 and 3A, were run on Dell desktop computers, running Windows 7. Stimuli were presented on 16 " CRT monitors set to $800 \times 600$ pixel resolution and a vertical blank interval of $10 \mathrm{~ms}(100 \mathrm{~Hz})$. Behavioral responses were recorded using standard USB keyboards.

The target and distractor stimuli were colored letters $\left(1.4^{\circ}\right.$ tall $)$ in the Arial Bold typeface. On each trial the stimulus display contained one red target letter $(r=251, g=$ $13, b=34, L=.256)$. Blue $(r=31, g=9, b=255, L=.123)$ and yellow $(r=204, g=$ 204, $b=0, L=.742$ ) distractor letters were present on some trials (see below). Each letter was presented in one of 6 positions on an imaginary circle with a radius of $5.5^{\circ}$, measured from the center of the fixation cross (white, $0.4^{\circ}$ ) to the center of each letter. The letters were masked by pattern masks, made up of a 10 by $10\left(2.7^{\circ}\right.$ by $\left.2.7^{\circ}\right)$ grid of colored squares. The masks were randomly chosen from a set of 10 images. The orientation of these were also randomized between $0,90,180$ and $270^{\circ}$, resulting in 40 different visual patterns in total. Following responses, the participants were presented with a green '+' (correct) or red '-' symbol (incorrect or "don't know" response) in the center of the screen $\left(0.6^{\circ}\right.$ wide $)$. 


\subsection{Procedure and Design}

The progression of a typical trial is illustrated in Fig. 1. Each trial started with a fixation cross in the center of the screen, of which the duration differed between experiments. Then an arousing stimulus was presented (see Method of Experiment 1A 1B, and 2). Next, 1 or 6 letters were presented for up to $120 \mathrm{~ms}$, depending on the current distraction condition. The letter identities were chosen randomly without replacement from the 26 letters of the English alphabet, so that all simultaneously presented letters had unique identities. Pattern masks covered all stimulus positions for $200 \mathrm{~ms}$, followed by a blank screen that was terminated by the participants' unspeeded response. Finally, feedback about performance on the current trial was present on screen for $1000 \mathrm{~ms}$, immediately followed by the next trial.

Throughout the experiment, the task was to report the identity of the red letter while ignoring the blue and yellow distractors. If participants thought they had identified the red target, they pressed the appropriate letter on the keyboard. If the target was completely missed (i.e., "don't know”), the participant was instructed to press the spacebar, rather than to make a blind guess.

A few times during each block, participants received additional feedback about the percentage correct of committed responses, i.e. not the accuracy overall, but the accuracy of responses when the participant committed to a certain letter, rather than giving a "don't know" response. Participants were instructed to strive towards a level of between 80 and $90 \%$ correct out of all committed responses. In addition, they received a 12 
message encouraging them to be more conservative if this percentage was below $80 \%$, that they were doing well if their performance was between 80 and $90 \%$ correct, and to be less conservative if their performance was above 90\% correct (adapted from Ásgeirsson, 2015; see also Kyllingsbæk, \& Bundesen, 2009).

\subsection{Bayesian Analyses}

Alongside traditional inferential statistics, we also used JASP (JASP team, 2016) to quantify relative evidence for competing explanatory models. JASP calculates Bayes factors or the relative probability of the current data, given a certain model, compared to another. Here, we use Bayes factors for their intuitive interpretation, and because they allow us to quantify evidence for - as well as against - a null hypothesis (see Jarosz \& Wiley, 2014, for some of the advantages of Bayesian statistics, and Matzke et al., 2015, for a recent example of their use in experimental psychology).

We ran the Bayesian repeated-measures ANOVA function of JASP (method of Bayesian analysis for factorial designs is described in Rouder et al., in press) on the data from all three experiments, and estimated Bayes factors for three relevant and competing models. All three models assumed a main effect of distractor condition: adding distractors to a display should negatively impact performance under all circumstances. This main effect is not of interest (nuisance factor; see Rouder et al., in press); the effects of interest are those related to the arousal manipulations and their potential interactions with distractor condition. Model $\mathrm{M}_{0}$ assumed that the arousal manipulation had no effect on performance. $M_{1}$, the first alternative model, assumed a main effect of arousal, but no 13 
interaction between arousal and distractor condition. If $\mathrm{M}_{1}$ provided the best account of the data, this would suggest that arousal affects visual processing, but that it does not modulate the effects of distraction. $\mathrm{M}_{2}$, the second alternative model, included a main effect of arousal as well as an interaction between arousal and distractor condition. If $\mathrm{M}_{2}$ provides the best account of our data, this would suggest that arousal modulates the effects of distraction level (i.e., competition), as predicted by $\mathrm{ABC}$ theory.

\subsection{TVA Modeling}

To enable more precise interpretation of our findings, we fitted mathematical models to the aggregated datasets (cf. Ásgeirsson, Kristjánsson, \& Bundesen, 2014) from Experiments 1A, 2 and 3A. The models were based on Bundesen's (1990) TVA. ABC theory predicts that aroused participants will be more selective (i.e., favor processing high-priority stimuli), and a major benefit of TVA models is that they can be used to quantify selectivity, while controlling for changes in other parameters such as overall

processing speed (i.e., evidence-accumulation rate) and the sensory threshold, which may be affected simultaneously by a given experimental manipulation. Therefore, the selective bias in arousal-biased competition should be captured by the selectivity parameters of TVA, and support more precise interpretations of the data by controlling for potential effects on other cognitive parameters. The details of the TVA models are explained in Appendix A. 


\title{
3. Experiment 1A - Phasic Emotional Arousal by IAPS
}

\author{
Pictures
}

\subsection{Method}

\subsubsection{Participants}

Twenty-eight participants (22 female), aged $18-27$ years old ( mean $=22.3, \mathrm{SD}=2.6$ ), participated in this experiment.

\subsubsection{IAPS pictures}

The arousing stimuli in Experiment 1 were 72 neutral, 36 pleasant and 36 unpleasant pictures from the IAPS (Lang et al., 2008). They were selected from the full set of IAPS pictures by considering their normative arousal and valence ratings (Fig. 2), as well as their consistency in normative ratings between male and female raters.

For the purposes of this experiment, the two arousing categories were collapsed into a single category. This was justified by a repeated-measures ANOVA showing that there was no main effect of picture category (pleasant or unpleasant) on performance $(F(1,27)=.55, p=.46)$, nor was there an interaction between picture category and distraction level $(F(2,54)=.24, p=.78)$. A Bayesian analysis supported this conclusion. A null model, with distractor condition as the single explanatory factor was estimated to give a better account of the data than one including a main effect of picture category (BF $=4.6$ for the null model), which was, in turn, more likely than a model including an 
interaction between the two factors ( $\mathrm{BF}=8.7$ for the main effects only model). A full list of the presented IAPS pictures can be found in Appendix B. Finally, we selected 20 clipart images of inanimate objects that were used to precede practice trials. These pictures depicted non-threatening objects, such as houses, tools, toys and kitchen appliances.

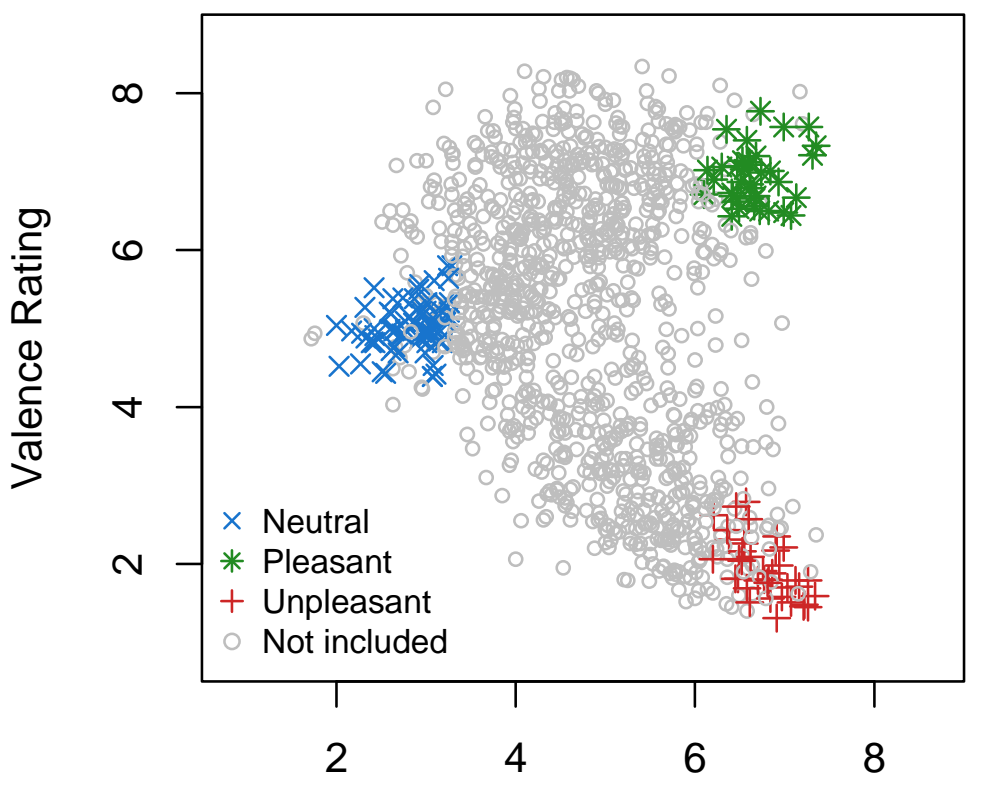

Arousal Rating

Fig. 2. All IAPS pictures plotted by arousal rating and valence rating from Lang et al. (2008). The three colored clusters represent the pictures in each of the three categories used in the current study.

The dimensions of the pictures were $13.7^{\circ}$ by $10.3^{\circ}$ (Fig. 1.8). On each trial, a picture was immediately followed by a 13.7 by $13.7^{\circ}$ achromatic random noise mask (Fig. 1.9), the 
purpose of which was to minimize the potential effects of afterimages and other sources of variance in sensory processing, in the interval leading up to target presentation.

\subsubsection{Procedure and Design}

The experiment consisted of four blocks of 144 trials each. A fixation cross was presented for 4 seconds, followed by an IAPS picture for 1 second. The long fixation period matched that of Sutherland and Mather (2012), and was deemed appropriate to minimize spillover of arousal effects across subsequent trials. Note, however, that several papers report clear behavioral effects in experimental designs with intermixed neutral and arousing IAPS pictures, and intertrial intervals that are shorter than in the current study (e.g., Koster, Crombez, Verschuere, \& De Houwer, 2004; Kristjánsson et al., 2012; Lee et al., 2012). Half of the pictures belonged to the neutral picture category, while the other half displayed arousing scenes. The pictures were followed by a 500-ms presentation of a noise mask, before a second fixation period, the duration of which was jittered randomly between 700 and $1300 \mathrm{~ms}$ (rounded to the nearest vertical blank of the $100 \mathrm{~Hz}$ monitor) until the letter stimuli appeared, resulting in an interstimulus interval (ISI; time from picture offset to letter onset) of $1200-1800 \mathrm{~ms}$. Following this period, a trial proceeded as explained in General Method and in Fig. 1. Target alone displays were presented for 20, 30, 50, 80 and $120 \mathrm{~ms}$. Distractor displays were presented for 50 and $80 \mathrm{~ms}$. Feedback about adherence to the desired response criterion was provided every 48 trials (see General Method). 


\subsection{Results}

Twenty-seven of the participants performed 576 trials of the experimental task, one block of data was lost for one participant due to a technical error. Extreme outliers in response times (mean +3 standard deviations of individual participants) were removed from the data, resulting in $1.48 \%$ data loss. To enable direct comparisons between the three distractor conditions, we limited the analysis to trials with exposure durations of 50 and $80 \mathrm{~ms}$. The remaining trials from the target alone condition were only included for the purposes of modeling (see Model Fits).

Fig. 3A shows the data from all experimental conditions, aggregated over all participants. A repeated-measures ANOVA comparing distractor conditions and picture category revealed no main effect of picture category $\left(F(1,27)=.17, p=.68, \eta_{\mathrm{p}}^{2}<.001\right)$, but a large effect of distractor condition $\left(F(2,54)=204.00, p<.001, \eta_{\mathrm{p}}{ }^{2}=.883\right)$. In all experiments, Greenhouse-Geisser corrections were applied whenever the assumption of sphericity was violated; in such cases, uncorrected degrees of freedom are reported. Importantly, there was no indication of an interaction between the two factors $(F(2,54)=$ $\left..08, p=.92, \eta_{\mathrm{p}}^{2}=.003\right)$.

Bayes factor comparisons strongly favored model $\mathrm{M}_{0}$, the model that only assumed a main effect of distractor condition. The observed data were estimated 5.6 times more likely under $\mathrm{M}_{0}$ than under $\mathrm{M}_{1}$, and more than 10 times more likely under $\mathrm{M}_{1}$ than under $\mathrm{M}_{2}\left(\mathrm{BF}_{01}=.096\right)$. This provides strong evidence (see Jeffreys, 1961, for the grouping scheme of Bayes factors followed here) against the prediction of ABC theory that the effects of competition are modulated by arousal. 
A major difference between the current study and the one reported by Sutherland and Mather (2012) is that their study consisted of only 40 experimental trials, while we used a more complex design with 576 trials and 144 pictures, each displayed four times. This might have led to a habituation effect that disguised early effects of arousal (but see Codispoti, Ferrari, \& Bradley, 2006). Therefore, we re-ran the repeated-measures ANOVA with block $(1-4)$ added to the factorial design. There was a main effect of block $\left(F(3,78)=11.33, p<.001, \eta_{\mathrm{p}}^{2}=.303\right)$, indicating poorer performance in block 1 than in blocks 2-4 (difference between 5.9 and 7.6\%; $t \mathrm{~s}>4.0$, Bonferroni-corrected $p \mathrm{~s}<$ .001). Importantly, the interaction terms that included picture category and block were not significant $\left(F_{\mathrm{s}}<1.6, p \mathrm{~s}>\right.$.19). Therefore, we have no evidence to suggest that arousal effects may have been present in the early stages of the experiment, but later disguised by habituation.
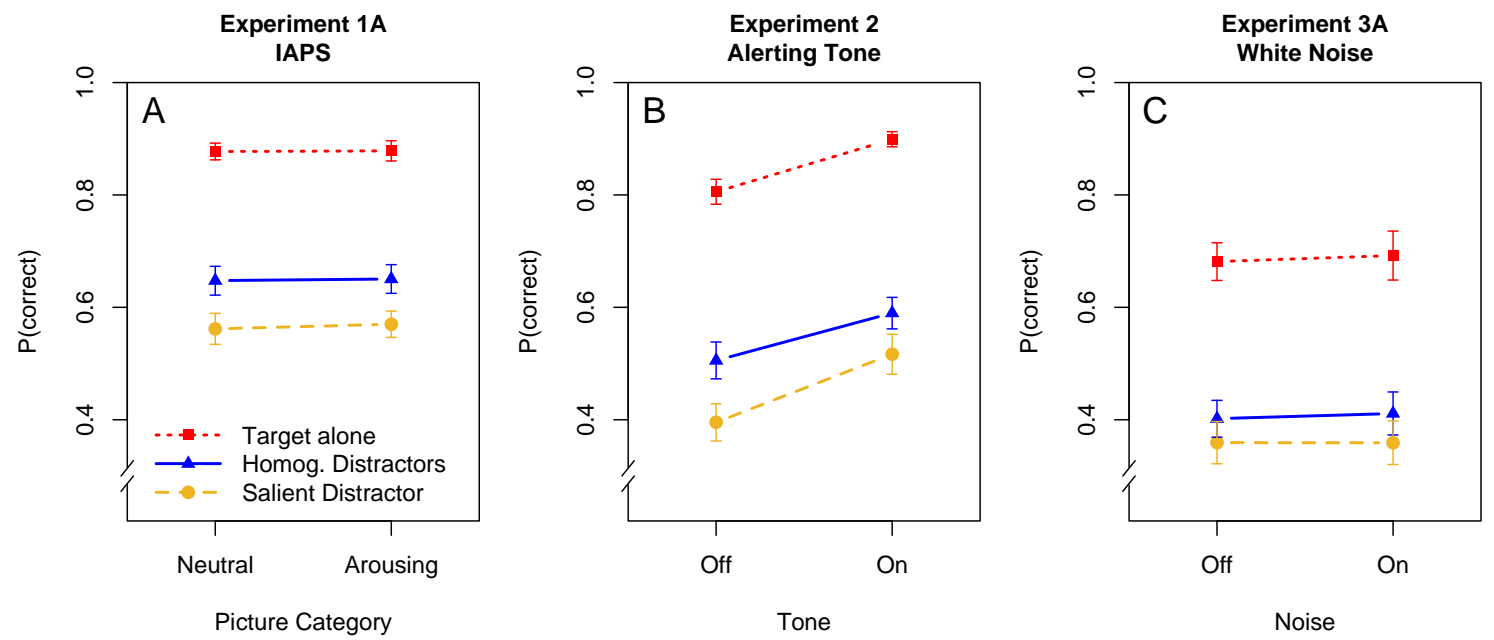

Fig. 3. The primary results from Experiments 1A, 2 and 3A. Error bars show \pm 1 SEM. Note that betweenexperiment comparisons are complicated by minor differences in experimental parameters, such as duration of pre-stimulus fixation and exposure durations. See the Method sections of each experiment for details. 
We did not find any behavioral effects of the arousal manipulation, raising the question whether the arousal manipulation was successful. Therefore, we ran a control experiment to examine whether the selected subsets of pleasant and unpleasant pictures had indeed aroused participants. IAPS pictures that are rated as highly arousing - pleasant or unpleasant - are known to cause sympathetic responses, such as increased pupil dilation and skin conductance (Bradley, Miccoli, Escrig, \& Lang, 2008), and have repeatedly been demonstrated to elicit a large late positive potential (LPP) component in eventrelated potential (ERP) research (e.g., Cuthbert, Schupp, Bradley, Birbaumer, \& Lang, 2000; Brown, van Steenbergen, Band, de Rover, \& Nieuwenhuis, 2012).

We recorded EEG from a new sample of participants, while they performed the task from Experiment 1A, to ensure that we were able to replicate the arousal-associated LPP modulation, and to test if this modulation lasted until the presentation of the letter display. We would consider this physiological evidence of a successful arousal manipulation.

\section{Experiment 1B - IAPS-evoked late positive potential as a marker of physiological arousal}

\subsection{Method}

\subsubsection{Participants}

Six participants $(5$ female), aged $21-24($ mean $=21.8)$ performed the experimental task. 20 


\subsubsection{Stimuli and Apparatus}

Stimuli and apparatus were identical to those in Experiment 1A, with the following exceptions. Stimuli were presented on a $60-\mathrm{Hz}$ LCD monitor, which forced a change in exposure durations (17, 33, 50, 83 and $133 \mathrm{~ms})$. The ISI was not jittered between 1200 ms and $1800 \mathrm{~ms}$, as in Experiment 1A, but was either $1200 \mathrm{~ms}$ (short ISI, 50\% of trials) or $1800 \mathrm{~ms}$ (long ISI, $50 \%$ of trials). This allowed us to examine whether the LPP modulation lasted throughout the ISI, while yielding a sufficient number of trials with the 1800-ms interval for getting a reliable ERP waveform. Finally, participants were connected to EEG-recording equipment throughout the experimental session.

\subsubsection{EEG Recording and Analysis}

EEG was recorded using a 64-channel BioSemi recording system. Recordings were limited to 17 scalp electrodes (F3, Fz, F4, C3, Cz, C4, CP3, CP1, CPz, CP2, CP4, P3, P1, $\mathrm{Pz}, \mathrm{P} 2, \mathrm{P} 4, \mathrm{Oz})$ and left and right mastoids. Horizontal (HEOG) and vertical eye movements (VEOG) were measured using bipolar recordings placed around the left eye. Offline processing was performed with the EEGlab toolbox (Delorme \& Makeig, 2004) for Matlab. The online sampling rate was $256 \mathrm{~Hz}$, but measurements were downsampled to $100 \mathrm{~Hz}$ offline. The data were filtered using a high-pass filter with a $0.03 \mathrm{~Hz}$ cut-off and a low-pass filter with $15 \mathrm{~Hz}$ cut-off. The data were split into 3700-ms long epochs, starting $500 \mathrm{~ms}$ before picture onset. Clear artifacts were rejected by visual inspection, leading to a data reduction of $1.2-15.2 \%$ (average 6.5\%) per participant. Eye-movement 21 
artifacts were removed using a Jader independent components analysis function in EEGlab. Finally, grand-average ERP waveforms were computed for each ISI (1200 or $1800 \mathrm{~ms}$ from IAPS offset) and preceding picture category (neutral or arousing). These averages were aggregated over three electrode channels in the centroparietal region (CP1, CPz, and CP2), where the LPP is known to be prominent (e.g., Brown et al., 2012;

Gerdes et al., 2013; Hajcak, MacNamara, \& Olvet, 2010).

\subsection{Results}



Fig. 4. Grand-average ERPs from the six participants in Experiment 1B. The large and protracted positivity following arousing pictures is clear in short (solid lines), and long (dotted lines) ISI trials, and remains significant in the windows of interest, immediately before target presentation. $*: p<.05$.

\subsubsection{Behavioral Results}


The behavioral results in this control experiment were in line with those of Experiment 1A. An analysis of the average performance for exposure durations 50 and $83 \mathrm{~ms}$ revealed a main effect of distractor condition $\left(F(2,10)=20.83, p<.001, \eta_{\mathrm{p}}{ }^{2}=.806\right)$, but no effect of picture category $\left(F(1,5)=.41, p=.55, \eta_{\mathrm{p}}^{2}=.075\right)$, and no interaction between the two factors $\left(F(2,5)=.90, p=.39, \eta_{\mathrm{p}}^{2}=.153\right)$.

\subsubsection{ERP results}

The grand-average ERP waveforms are shown in Fig. 4. The waveforms show a marked and prolonged LPP modulation that was largest during presentation of the IAPS picture and smaller during the presentation of the noise mask and subsequent fixation period. To test the difference in voltage after neutral and arousal pictures in the $100 \mathrm{~ms}$ leading up to target presentation, one-sided paired $t$-tests were performed. This LPP modulation was significant on short ISI trials $(-2.7 \mu \mathrm{V} ; t(5)=-2.61, p=.024, d=-.61, \mathrm{BF}=4.2)$, as well as on long ISI trials $(-3.7 \mu \mathrm{V} ; t(5)=-2.06, p=.047, d=-.48, \mathrm{BF}=2.5)$. These results suggest that the arousing pictures caused a robust LPP modulation, and that this physiological arousal effect was still ongoing at the time of target presentation.

\section{Experiment 2 - Alerting Tone}

\subsection{Method}

\subsubsection{Participants}


Twenty-eight participants (22 female), aged $18-30$ years old $($ mean $=23.1, \mathrm{SD}=3.1$ ), participated in this experiment.

\subsubsection{Stimuli and Apparatus}

Participants wore Sennheiser 202HD closed-back on-ear headphones for the duration of the experiment. On half of the trials, the letter presentation (Fig. 1) was preceded by an 800-Hz tone, played at $80 \mathrm{~dB}$ for $150 \mathrm{~ms}$ (Jepma, Wagenmakers, Band, \& Nieuwenhuis, 2009).

\subsubsection{Procedure and design}

The experiment was split into two blocks of 216 trials each. The experimental procedure is depicted in Fig. 1. A trial began with the presentation of a fixation cross for 1-3 seconds (jittered randomly to increase temporal uncertainty), followed by the onset of the letter display. On half of the trials a task-irrelevant tone was played $300 \mathrm{~ms}$ before the appearance of the letter display. On the other half, a silent sound object preceded the visual stimulus. Feedback on behavior was provided after every 56 trials. Other details are described in General Method.

\subsection{Results}

All 28 participants performed 432 trials of the experiment. Extreme outliers in response times (mean +3 standard deviations of individual participants) were removed from the data, resulting in $1.46 \%$ data loss. 
Fig. 3B displays the average performance in Experiment 2, showing a clear trend towards better performance when the alerting tone was present. A 3 by 2 repeatedmeasures ANOVA revealed main effects of distractor condition $(F(2,54)=159.9, p<$ $\left..001, \eta_{\mathrm{p}}^{2}=.856\right)$ and of tone presence $\left(F(1,27)=118.9, p<.001, \eta_{\mathrm{p}}^{2}=.815\right)$, but no interaction effect between the two factors $\left(F(2,54)=1.41, p=.25, \eta_{\mathrm{p}}{ }^{2}=.05\right)$. A followup ANOVA with block added as an additional factor yielded a main effect of block ( $F(1$, $\left.27)=20.26, p<.001, \eta_{\mathrm{p}}{ }^{2}=.429\right)$, but no interactions between block and the other factors ( $p$ 's $>.17)$. The follow-up analysis did not suggest that habituation to the alerting tone disguised an interaction.

A Bayesian analysis revealed the data to be most likely under model $\mathrm{M}_{1}$, which assumed main effects of distractor condition and tone presence but no interaction. $\mathrm{M}_{1}$ was estimated to be $1.4 * 10^{8}$ more likely than $\mathrm{M}_{0}$, and 6.0 times more likely than $\mathrm{M}_{2}$. These results suggest that there was extreme evidence for a (beneficial) main effect of arousal, and moderate evidence against an interaction between arousal and level of distraction.

\section{Experiment 3A - Tonic Arousal by White Noise}

\subsection{Method}

\subsubsection{Participants}

Twenty-one participants (13 female), aged $18-30$ years old $($ mean $=23.3, \mathrm{SD}=2.9)$, participated in this experiment. 


\subsubsection{Stimuli and Apparatus}

Participants wore Sennheiser 202HD closed-back on-ear headphones during all blocks of the experiment. On half of these blocks, no audio was played. On the other half, white noise with a peak intensity of $90 \mathrm{~dB}$ was played.

\subsubsection{Procedure and Design}

Participants performed 20 practice trials without white-noise stimulation before starting the experiment, which consisted of four blocks of 162 trials each. The order of blocks was counterbalanced, so that half of the participants were stimulated by noise in the first and third blocks, while the other half was stimulated in the second and fourth blocks (ABAB or BABA design). The progression of a typical trial is illustrated in Fig. 1. The fixation duration was a constant of $500 \mathrm{~ms}$. There was a mandatory 5-minute break between blocks to allow for noise-induced arousal to return to baseline levels.

\subsection{Results}

All 21 participants performed 648 trials of the experiment. Extreme outliers in response times (mean +3 standard deviations of individual participants) were removed from the data, resulting in $1.27 \%$ data loss.

Fig. 3.C shows the results from Experiment 3A. It is clear that the presence of white noise did not have much impact on performance. A 3 by 2 repeated-measures ANOVA revealed a strong main effect of distractor condition $(F(2,40)=206.5, p<.001$, 
$\left.\eta_{\mathrm{p}}{ }^{2}=.912\right)$ but no main effect of white noise $\left(F(1,20)=.15, p=.70, \eta_{\mathrm{p}}{ }^{2}=.007\right)$ or an interaction between the two factors $\left(F(2,40)=.15, p=.86, \eta_{\mathrm{p}}^{2}=.007\right)$.

Bayes factors estimates showed the data to be 4.6 times more likely under $\mathrm{M}_{0}$ than $\mathrm{M}_{1}\left(\mathrm{BF}_{01}=.216\right)$, suggesting that a main effect of white noise stimulation was unlikely. Furthermore, model $\mathrm{M}_{1}$ was 7.0 times more likely than $\mathrm{M}_{2}$, providing evidence against arousal-modulated competition.

Experiment 3A did not reveal any systematic differences in performance under white noise versus silence, raising the question whether the white-noise manipulation was successful in increasing arousal levels. Therefore, we ran a control experiment, in which we monitored pupil size while participants performed the same task. Pupil size is an established biological marker of tonic arousal and has been shown to track the activity of brainstem arousal centers such as the locus coeruleus (e.g., Joshi, Li, Kalwani, \& Gold, 2016; Murphy, O’Connell, O’Sullivan, Robertson, \& Balsters, 2014; Nieuwenhuis, de Geus, \& Aston-Jones, ,2011).

\section{Experiment 3B - Pupil Dilation under Tonic White Noise Stimulation}

\subsection{Method}

\subsubsection{Participants}


Eight participants ( 7 female), aged $20-30$ (mean $=24.6, \mathrm{SD}=3.2$ ) were recruited for this experiment. All reported normal color vision, and normal or corrected-to-normal vision.

\subsubsection{Apparatus}

Experiment 3B was identical to Experiment 3A, with the following exceptions. Stimuli were presented on a $60-\mathrm{Hz}$ LCD monitor, in a moderately lit room. This forced a change in exposure durations (17, 33, 50, 83 and $133 \mathrm{~ms})$. Pupil size was measured with an SR Research EyeLink 1000 eye tracker. Participants used a chin rest during the experiment.

\subsubsection{Procedure}

A 5-point calibration procedure was run before each block of trials. Participants were instructed to blink only during the presentation of feedback (Fig. 1.7), and the duration of the feedback screen was doubled ( 2 seconds) to make this instruction easier to follow. In other respects, the procedure was identical to that of Experiment 3A.

\subsubsection{Pupillometry}

To minimize confounds related to between-trial differences in luminance, response times and other sources of noise, we only analyzed pupil data from the 500-ms fixation interval between the feedback presentation of trial $n-1$ and target onset in trial $n$. Pupil area was recorded in terms of arbitrary pixels. The data from each trial of the experiment were checked for measurement artefacts. Trials with blinks and extreme rates of change $(<150$ pixels/ms) were excluded from analysis. Outliers, defined as trials with pupil values 
greater than \pm 3 standard deviations from the participant's average pupil area, were also discarded, resulting in $5.1 \%$ data loss. Finally, pupil diameter was calculated from pupil area, and averaged across trials. The resulting trial averages were $z$-scored at the subject level.

\subsection{Results}

The behavioral data from Experiment 3B were consistent with Experiment 3A. There was no main effect of white noise on letter identification $\left(F(1,7)=.86, p=.39, \eta_{\mathrm{p}}^{2}=.109\right)$, and no interaction between distractor condition and white noise $(F(2,14)=.97, p=.40$, $\left.\eta_{\mathrm{p}}^{2}=.121\right)$.

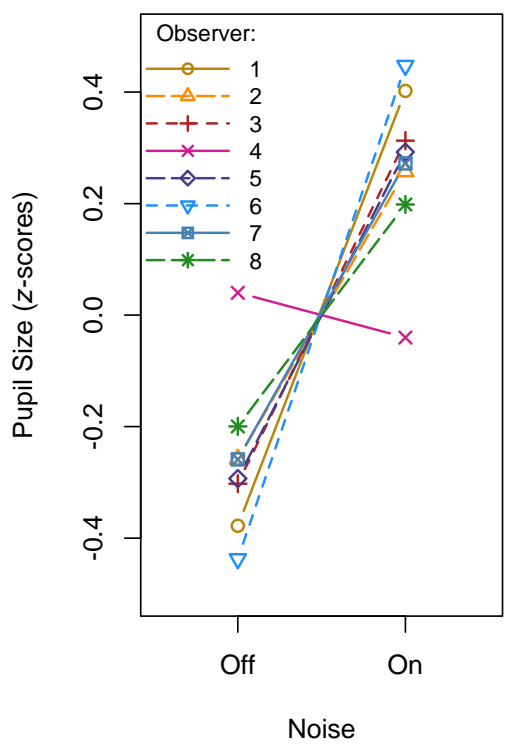

Fig. 5. Results from Experiment 3B. Data points show the mean pupil diameters ( $z$-scored within-subject) after controlling for time-on-task effects. 
To assess whether pupil size was affected by the presence of white noise, we started by regressing out typical time-on-task effects (van den Brink, Murphy, \& Nieuwenhuis, 2016; Knapen et al., 2016). This was done with simple linear regression on each participant's data, with trial number as the dependent variable and pupil size as the independent variable. The average value of the residuals was calculated separately for each of the eight participants (see Fig. 5), and separately for noise-present and noiseabsent blocks. These averages were submitted to a paired-samples $t$-test. The test revealed a significant difference between the blocks in which white noise was present and blocks in which it was absent $(t(7)=5.16, p=.001, d=-3.64, \mathrm{BF}=65.9)$. This result suggests that most participants in Experiment $3 \mathrm{~A}$ were in a state of increased arousal while performing the task under white-noise stimulation. Yet, in both Experiments 3A and 3B, behavior was not affected by this arousal manipulation.

\section{Model Fits}

The Bayesian and traditional frequentist analyses reported above were performed without any assumptions about the interplay between the different cognitive mechanisms required to successfully perform the singleton letter identification task. However, the wide range of target exposure durations enabled us to fit TVA models to the aggregated data from each experiment (Bundesen, 1990). Based on the theoretical assumptions of TVA, these models were able to separate the contributions of distinct parameters representing the participants' selectivity, while controlling for potential differences between conditions in 
overall processing speed and sensory threshold. The details of these TVA models are described in Appendix A.

The model parameters of primary interest were the attentional weights, which represented the amount of attention allocated to a given stimulus type (target, blue distractor or yellow distractor) in a given arousal condition. ABC theory posits that arousal changes the division of attentional resources among high- and low-priority stimuli, biasing the competition in favor of high-priority stimuli. When translated into the terminology of TVA, ABC theory predicts that arousal should reduce the weight of lowpriority blue distractors, relative to the weights of the high-priority targets. Thus, ABC theory predicts that in the homogeneous distractor condition arousal should increase the proportion of attentional resources allocated to the target, where the proportion of attentional resources refers to the division of all available resources represented by the $s$ parameter of TVA, which is an indirect measure of processing speed (see Equation 3, Appendix A). For the sake of simplicity, we will refer to this as processing speed throughout. Likewise, in the salient distractor condition arousal should increase the sum of the proportional resources allocated to targets and salient distractors (i.e., both highpriority stimuli). Conversely, in both conditions arousal should decrease the proportion of attentional resources allocated towards blue distractors.

Another question is whether arousal level affects overall processing speed, another TVA parameter, which reflects the total sum of available resources. In a recent theoretical paper, Bundesen, Vangkilde, and Habekost (2015) proposed a multiplicative relationship between arousal level and processing speed (equation 4, p. 118). One of the 31 
predictions that follow directly from this proposal is that an increase in arousal should lead to a monotonic rise in processing speed. Consistent with this prediction, previous TVA studies have yielded evidence of processing speed enhancements with increased temporal attention (Matthias et al., 2010; Sørensen et al., 2015; Vangkilde, Coull, \& Bundesen, 2012; Vangkilde et al., 2013). In addition, Bundesen et al. proposed that the influence of arousal manipulations on selectivity (i.e., the division of available resources) follows an inverted-u-shaped function. We tested these two predictions by examining the effects of arousal on the TVA parameters corresponding to processing speed and selectivity.

Fig. 6A-C show the fits of a 7-parameter TVA model to the empirical results from each of our main experiments. The modeling procedure yielded parameter estimates of the processing speed (s) and the weight of blue ( $\left.w_{\text {blue }}\right)$ and yellow ( $\left.w_{\text {yellow }}\right)$ distractors, which were left free to vary between the arousing and neutral conditions, and a single parameter estimate of the sensory threshold $\left(t_{0}\right)$. Table 1 provides the exact parameter estimates of the 7-parameter model fits. 
A

IAPS

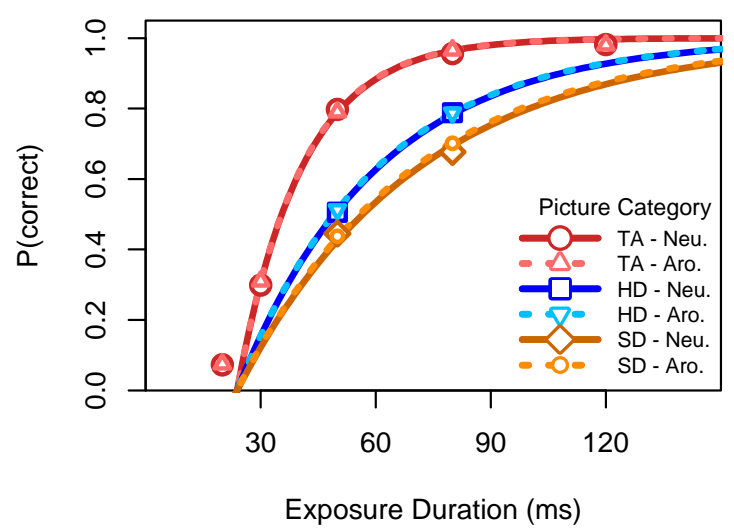

C

White Noise

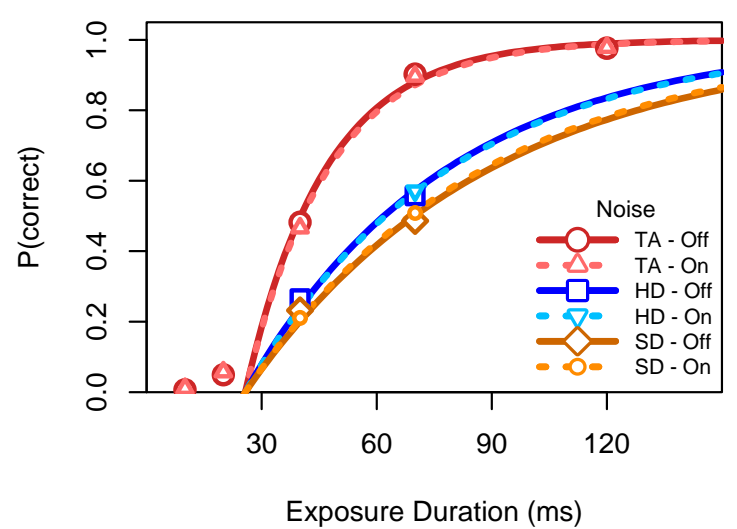

B

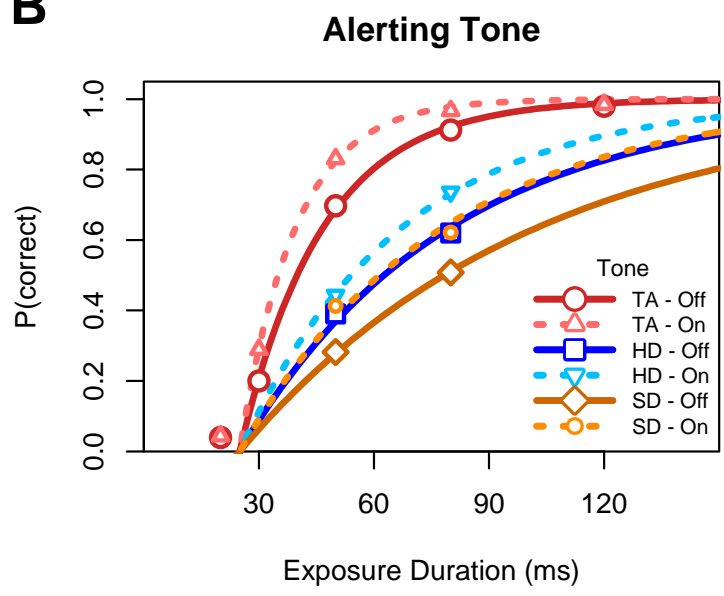

D Proportional Allocation of Attention

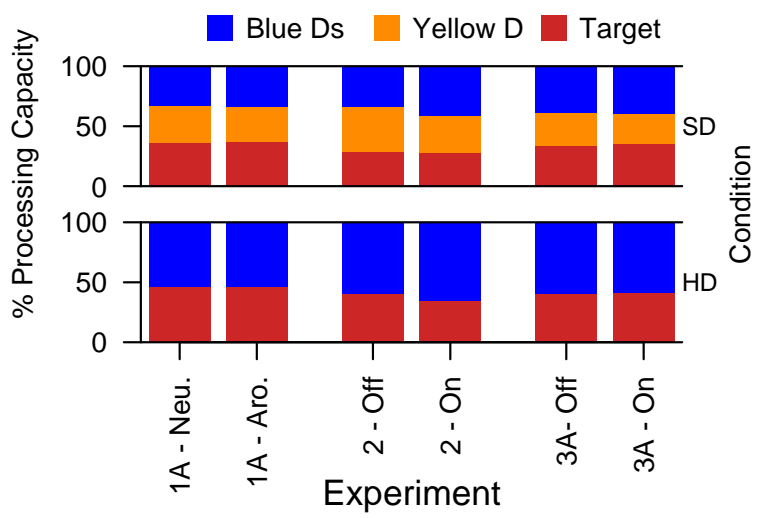

Fig. 6. A-C: Empirical (points) and simulated performance (lines) of a 7-parameter TVA model, which was fitted to the data collapsed across all participants. Distraction levels are abbreviated as TA: target alone, HD: homogeneous distractors, and SD: salient distractor. D: The relative allocation of processing resources for each stimulus type, as estimated by the TVA model. Note that the blue area of the bars represents 4 blue distractors in the SD condition, but 5 blue distractors in the HD condition. 
Table 1. TVA parameter estimates from a 7-parameter model of the data from Experiments 1A, 2 and 3A.

\begin{tabular}{|c|c|c|c|c|c|c|}
\hline \multirow[b]{2}{*}{ Parameter } & \multicolumn{2}{|c|}{ IAPS } & \multicolumn{2}{|c|}{ Alerting Tone } & \multicolumn{2}{|c|}{ White Noise } \\
\hline & Neutral & Arousing & Off & On & Off & On \\
\hline Processing speed $(s)$ & 59.4 & 59.8 & 46.4 & 69.3 & 48.0 & 46.3 \\
\hline Weight of blue distractor ( $w_{\text {blue }}$ ) & .235 & .235 & .304 & .381 & .300 & .289 \\
\hline Weight of yellow distractor ( $w_{\text {yellow }}$ ) & .866 & .814 & 1.352 & 1.119 & .844 & .716 \\
\hline Threshold for visual perception $\left(t_{0}\right)$ & \multicolumn{2}{|c|}{23.8} & \multicolumn{2}{|c|}{25.0} & \multicolumn{2}{|c|}{25.7} \\
\hline$R^{2}$ & \multicolumn{2}{|c|}{.986} & \multicolumn{2}{|c|}{.994} & \multicolumn{2}{|c|}{.993} \\
\hline
\end{tabular}

The close correspondence between empirical and simulated performance

displayed in Fig 6A-C, as well as the $R^{2}$ values (calculated by using the ordinary least squares method) in Table 1 indicate that the 7-parameter model provides an excellent account of the empirical data. Importantly, in contrast to the predictions of ABC theory and Bundesen et al. (2015), Table 1 indicates that arousal level did not have a robust effect on attentional weights (i.e., selectivity). If anything, the changes in weights were in the opposite directions from those predicted. Furthermore, processing speed was increased by the presence of an alerting tone (Experiment 2), but not affected by arousing pictures and white noise (Experiments $1 \mathrm{~A}$ and $3 \mathrm{~A}$ ). Thus, the results provide mixed evidence for the prediction of Bundesen and colleagues that arousal has a multiplicative effect on processing speed. These observations were supported by the following model comparisons: (i) For Experiments 1A and 3A, a 4-parameter model, in which none of the parameters were left free to vary between arousing and neutral conditions, had almost the same goodness of fit as the 7-parameter model $\left(\Delta R^{2}<.001 ; F \mathrm{~s}(3,11)<.1 ; p \mathrm{~s}>.97\right)$; (ii) For Experiment 2, a 5-parameter model, in which only processing speed was allowed to 34 
vary with arousal level, had almost the same goodness of fit as the 7-parameter model $\left(\Delta R^{2}<.01 ; F(2,11)=.64 ; p=.60\right)$, whereas the 4-parameter model, which did not vary by arousal at all, explained significantly less variance than the 7-parameter model $\left(\Delta R^{2}=\right.$ $3.2 ; F(3,11)=19.3 ; p<.001)$.

Our key finding that arousal did not modulate the division of attentional resources is highlighted in Fig. 6D, in which attentional weights have been plotted as proportions of the available resources. None of the experiments show an arousal-induced bias in resource allocation towards the target (homogeneous distractor condition) or target and salient distractor (salient distractor condition). The TVA modeling results therefore corroborate the conclusion that arousal-biased competition did not occur in our focusedattention task.

Here, we have presented models fitted to aggregated data (cf. Ásgeirsson, Kristjánsson, \& Kristjánsson, 2014). The conclusions drawn from the group-level models were corroborated by subject-level models (presented in Appendix C. )

\section{General Discussion}

The goal of this research was to investigate how arousal affects attentional preferences in visuospatial attention. In particular, we tested key predictions of a prominent and attractive theory, ABC theory (Mather, \& Sutherland, 2011; Mather et al., 2016), which attempts to explain a large and seemingly contradictory literature in experimental 
psychology and neuroscience, by proposing that the effect of arousal on perception and memory depends on the priority of the signals being processed at a given time. We presented a target of fixed color and intensity, but manipulated the surrounding distractor stimuli to create different levels of competition in visual processing. Arousal level was successfully manipulated in three different ways, as evidenced by ERP modulations in response to emotionally arousing pictures (Experiment 1B), improvements in stimulus processing speed after the presentation of alerting auditory tones that are known to elicit a phasic arousal response (Experiment 2; Hackley \& Valle-Inclán, 2003; Jepma et al., 2009; Tona et al., 2016), and by large increases in pupil diameter under white-noise stimulation (Experiment 3B). Two of these manipulations affected trial-to-trial changes in (phasic) arousal, one manipulation affected arousal in a blockwise (tonic) fashion.

If the principles of arousal-biased competition apply in the domain of focused visuospatial attention, then $\mathrm{ABC}$ theory predicts that the effect of competition will be modulated by the participant's level of arousal. However, we found no evidence for such arousal-biased competition in any of the experiments. Traditional statistical analyses failed to demonstrate the crucial interaction between the effects of arousal level and distractor manipulations. These analyses were followed up using Bayesian statistics to quantify the evidence for and against arousal-biased competition in our task. Bayes factors revealed strong (Experiment 1) to moderate (Experiments 2 and 3) evidence against arousal-biased modulations of attention. In fact, arousal only affected task performance when arousal was manipulated by an auditory alerting tone (Experiment 2), but this effect was independent of competition with surrounding distractors. 36 
A TVA model was fitted to the data to isolate the model parameters specifically related to attentional competition, while controlling for other factors, such as differences in processing speed and sensory threshold (Bundesen, 1990). The TVA model fits and model comparisons corroborated the outcomes of the frequentist and Bayesian statistics: Increased arousal did not increase attentional weights associated with high-priority stimuli and did not decrease attentional weights associated with low-priority stimuli. Altogether, these findings provide a challenge for (the scope of) ABC theory.

There are several differences between our focused-attention experiments and the divided-attention experiments of Sutherland and Mather (2012), which did yield evidence for arousal-biased competition. One prominent difference is that our experiments consisted of far more trials (e.g., Experiment 1: 576; Experiment 2: 432) than Sutherland and Mather's (40 trials). However, we found that time-on-task did not interact with the main and interaction effects of arousal, ruling out the possibility that arousal-biased competition was evident in the first phase of the experiments but then diminished because of habituation to the arousing pictures or alerting tones. A second noticeable difference is that our experiments had roughly half the number of participants as Sutherland and Mather's, raising the question whether we might have found evidence for arousal-biased competition with a larger sample size. However, our use of Bayesian statistics allowed us to compute evidence for and against the key prediction of $\mathrm{ABC}$ theory, and as noted above the Bayes factors revealed strong (Experiment 1) to moderate (Experiments 2 and 3) evidence against arousal-biased competition. These findings, as well as the TVA 
parameter values and model comparisons, rule out a lack of statistical power as an explanation for the discrepancy in results.

A third and essential difference is that in Sutherland and Mather's task, stimulus priority was fully based on a bottom-up factor (salience in terms of visual contrast) whereas in our experiments stimulus priority was based on both a bottom-up factor (salience in terms of color) and a top-down factor (task relevance: target versus distractors). According to $\mathrm{ABC}$ theory arousal should amplify the effects of both types of influences, and Mather and colleagues have reported evidence that arousal amplifies the effects of top-down priority in memory formation when the arousal response occurs briefly after the to-be-memorized stimulus. However, in recent work Mather and colleagues (Mather et al., 2016, pp. 16; Sutherland, McQuiggan, Ryan, \& Mather, 2017) have proposed that an arousal response that occurs briefly before stimulus presentation may not elicit arousal-biased competition effects because arousal impairs top-down prioritization, as a result of which there are no highly activated representations to amplify. This proposal is, in part, based on findings that high stress impairs prefrontal cortex function (Arnsten, 2000), and hence on the tentative assumption that those findings generalize to the moderate levels of arousals examined in the current research domain. Preliminary evidence for this assumption was obtained in a study reporting that viewing emotional pictures reduced top-down overt attention (as measured by viewing time) to subsequently presented task-relevant stimuli while increasing attention to taskirrelevant stimuli, at least for one stimulus category (Sutherland et al., 2017). Although this proposal of Mather and colleagues requires further empirical support, it can possibly 38 
account for the absence of arousal-biased competition effects in our study, given the strong top-down factor in our three experiments. Maybe the bottom-up prioritization associated with stimulus salience was not strong enough in itself to cause arousal-biased competition effects. It must be noted, however, that the target was highly salient in the homogeneous distractor condition, it being a color singleton, as well as the item of highest luminance.

Perhaps the critical difference between the two studies concerns the involvement of learning or memory, processes that have long been known to be modulated by arousal (Eysenck, 1976; Mather, 2007). Sutherland and Mather (2012 see also Sørensen et al., 2015) used a whole-report procedure, which required participants to attend to and encode in visual short-term memory as many of the eight letters in the target display as possible. In contrast, in our task participants were required to identify only one (red) target letter, to be reported at the end of the trial, thus imposing a minimal memory load. This raises the question of whether arousal biases competition in perception in the absence of a nontrivial learning/memory component (Bacon, 1974). Some of the strongest evidence for arousal-biased competition comes from memory (Sakaki et al., 2014) and perceptual learning (Lee et al., 2012) studies. In contrast, Lee et al. (2012) did not find arousalbiased competition in visual search, a perceptual task. Although there is plenty of evidence for positive or negative main effects of arousal on perceptual sensitivity, future research should address under which circumstances purely perceptual tasks show the characteristic "winner-take-more/loser-take-less" dynamics that are indicative of arousalbiased competition. 
Our study also allowed us to test the predictions of an alternative theory about the effects of arousal on components of cognition (Bundesen et al., 2015). One assumption of this theory is that attentional selectivity (or competition) is related to arousal according to an inverted-u-shaped function: attentional selectivity should be best at intermediate levels of arousal. Strictly taken, our binary manipulation of arousal level does not allow us to identify a curvilinear relationship between arousal and selectivity. That is, our null results can in principle be explained by this theory by assuming that, on average, the low- and high-arousal conditions placed the participants at points to the left and right of the curve's optimum that were associated with similar selectivity. However, we think it is unlikely that this scenario occurred in all three of our experiments. In addition, our TVA modeling results also revealed no consistent effect of arousal on the attentional weights of highand low-priority stimuli, the model parameters that together determine attentional selectivity. Therefore, our results do not seem to support the selectivity assumption of Bundesen and colleagues. Nonetheless, definitive validation of the assumption awaits a more rigorous test, involving the manipulation of arousal over multiple levels (see Vangkilde et al., 2013).

The second assumption of Bundesen et al.'s (2015) theory is that increasing arousal should lead to a monotonic rise in overall processing speed. We did not find evidence for such a rise after presentation of emotionally arousing pictures or during intense white-noise stimulation, but only when arousal was manipulated using an auditory cue, which provided information about the onset of the target display (300 ms later; Lawrence \& Klein, 2013). This latter finding is consistent with previous studies that 40 
found an effect of phasic auditory alerting and temporal uncertainty on TVA's processing speed parameter (Petersen, Petersen, Bundesen, Vangkilde, \& Habekost, 2017; Wiegand, Petersen, Lasner, Finke, \& Habekost, 2016). In contrast, the null findings in Experiments 1 and 3 provide a challenge for the TVA account of arousal effects on cognition, but also a more general challenge, because they underscore the fact that very little is known about the relationship between different manipulations of arousal. To what extent do these manipulations affect the same system (Hanoch \& Vitouch, 2004; Robbins, 1997)? Is there evidence for a unidimensional arousal construct, an emotional arousal state that can only differ in intensity, or can arousal states stem from different sources (Calderon, Kilinc, Maritan, Banavar, \& Pfaff, 2016; Hanoch \& Vitouch, 2004)? Should theories of arousal and cognition, such as ABC theory and TVA, differentiate between the various ways in which arousal can be manipulated? We hope that our study provides useful constraints for the further development of these theories. Finally, although we used wellestablished manipulations of arousal, and report successful manipulation checks, it is possible that we would have found more support for these theories with stronger arousal manipulations.

\section{Acknowledgements:}

The authors thank Saskia Heijnen and Rudy van den Brink for their contributions to data collection and EEG analysis, respectively, and Mara Mather, Christian H. Poth, Werner X. Schneider, Thomas A. Sørensen, and an anonymous reviewer for helpful comments on 
an earlier draft of this paper. The research was supported by an ERC consolidator grant awarded to S. Nieuwenhuis.

\section{Appendix A. An explanation of TVA models}

The central equations of TVA describe how limited attentional resources are divided amongst all objects in a visual scene. According to TVA, the selection and recognition of a visual element can be described as the results of solving two very similar decision problems. The first decision problem takes the form "object $x$ has feature $j$ ” where $x$ is any visual object and feature $j$ is a feature of some importance to the observer in the context of current behavior. This process is called filtering, and its solution results in an attentional weighting of a visual object $x$. This is formalized in the weight equation of TVA:

$$
w_{x}=\sum_{j \in R} \eta(x, j) \pi_{j}
$$

where $R$ is the set of all visual features, $\eta(x, j)$ is the strength of the evidence for object $x$ having feature $j$, and $\pi_{j}$ is the current importance of category $j$ (Bundesen, 2008, p. 6061). The attentional weights comprise the selective term in the rate equation of TVA-that 
is, how limited resources are divided between visual objects. The rate of processing of a given element $x$ is given by the rate equation of TVA:

$$
v(x, i)=\eta(x, i) \beta_{i} \frac{w_{x}}{\sum_{z \in S^{w_{z}}}}
$$

where $\eta(x, i)$ is the strength of evidence for object $x$ belonging to report category $i, \beta_{i}$ is the perceptual bias towards making categorization $i, w_{x}$ is the attentional weight of object $x$, and $S$ is the set of all visual objects in the display. The product of the strength of evidence for element $x$ belonging to category $i$, and the perceptual bias for making that categorization, is collectively represented by the processing speed parameter $(s)$. In the current study, we never systematically manipulate the sensory evidence of targets (they are always of the same color and brightness), and we can, therefore, assume the $\eta(x, i)$ to be constant throughout. Conversely, arousal may affect perceptual bias $\left(\beta_{i}\right)$, as hypothesized by Bundesen et al. (2015). Therefore, we can simplify the terms $\eta(x, i) \beta_{i}$ into the processing speed parameter $s$, and deduce that if an arousal manipulation affects perceptual bias in our experiments, this will be evident as a modulation of the $s$-parameter in our models. Here, the $s$-parameter represents basic sensory effectiveness of the stimulus and serves as an indirect measure of processing speed. The method of estimating processing speed indirectly with the $s$-parameter is adapted from Duncan et al. (1999; p. 452-453). Processing speed is, in turn, defined as the sum of all processing rates(see Bundesen \& Habekost, 2008, p. 65). The simplified rate equation 


$$
v(x)=s \frac{w_{x}}{\sum_{z \in S} w_{z}}
$$

highlights the two currently critical processes: firstly, the $s$-parameter that is modulated by arousal but does not directly affect selectivity, according to Bundesen et al. (2015); secondly, the division of the available resources (the weight ratio), predicted by ABC theory to be biased towards high-priority stimuli under arousal (Mather \& Sutherland, 2011).

\section{Example:}

In our study, we included conditions with three levels of target selection difficulty. The specific conditions are depicted in Fig. A1, alongside a plot of three encoding functions in TVA. For the purpose of illustration, we hand-selected plausible values for the free parameters: processing speed $(s)$, the attentional weights of the two types of distractors

relative to a target, and the threshold for visual perception $\left(t_{0}\right)$. This threshold anchors an exponential function to an intercept of the $\mathrm{x}$-axis, and represents minimum effective exposure duration (Bundesen \& Habekost, 2008, p. 58).

A simple model of performance in one of the reported experiments may be understood through examples of the situations caused by the manipulation of distractor 
number and type.

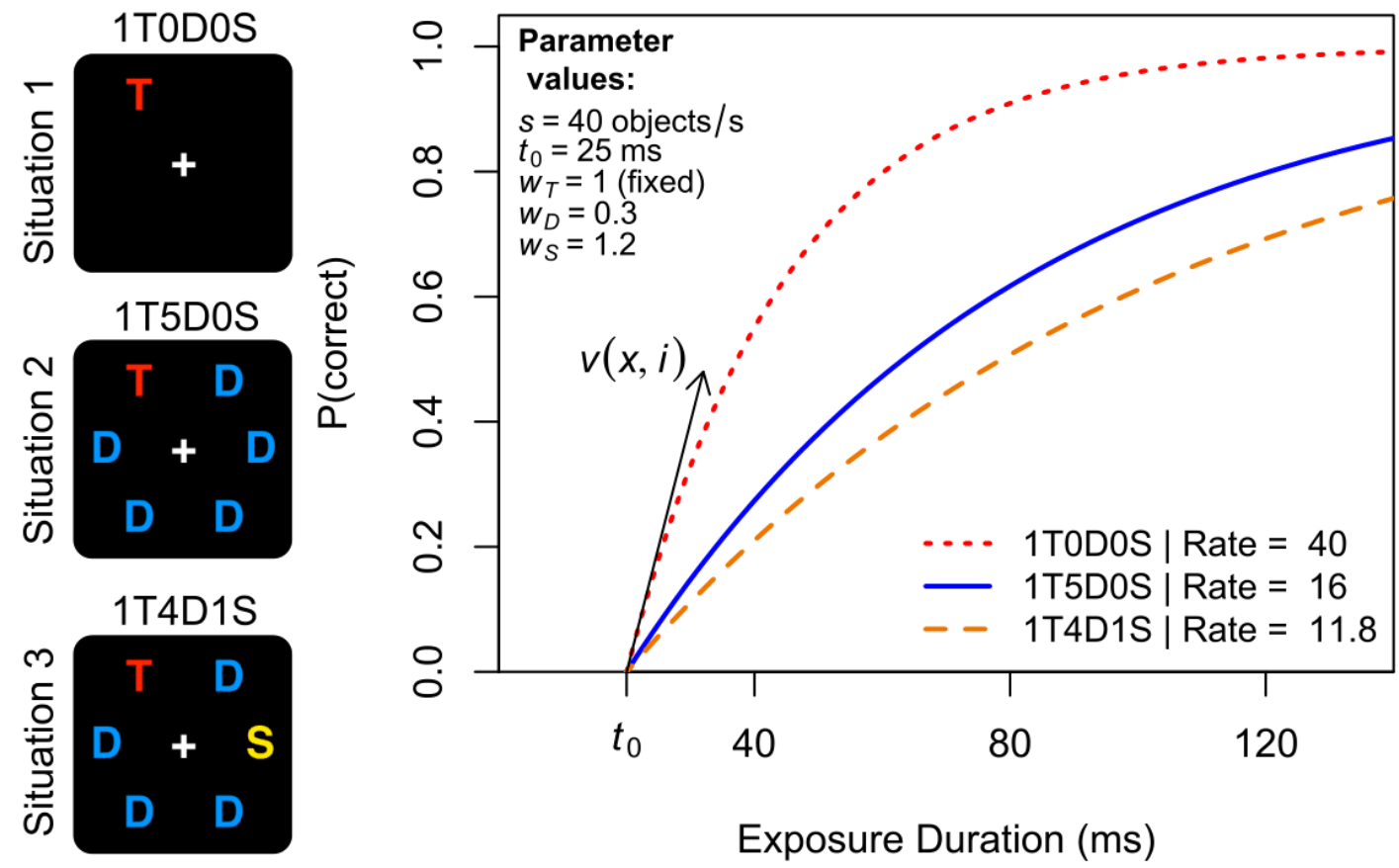

Fig. A1. Left: Schematic representations of the three types of distraction levels presented in the experiments. Right: Probability of having encoded a target under each of the three distraction levels, given the specified TVA-parameter values.

The first situation is the target alone condition, or 1T0D0S, which represents the number of targets (T), regular blue distractors (D) and salient distractors (S) in the display. To calculate the rate of target processing in this situation, we apply equation 3 with the given parameter values and obtain

$$
v=40 \times \frac{1}{(1 * 1)+(0 * .3)+(0 * 1.2)}=\frac{40}{1}=40.0
$$


The rate of target processing in situation 1 is equal to the overall processing speed, because the target is not flanked by distractors, and therefore all available attentional resources may be deployed to the target. A theoretical encoding function describing processing in situation 1 is represented by the red line in Fig. A1.

Situation 2 - the homogeneous distractor condition - introduces demands on the observers' ability to select targets from distractors. By applying the simplified rate equation we get

$$
v=40 \times \frac{1}{(1 * 1)+(5 * .3)+(0 * 1.2)}=\frac{40}{2.5}=16.0
$$

This time the rate of target processing has dropped because attentional resources are also deployed to distractors. Each blue distractor receives $30 \%$ of the amount of attentional resources deployed to a given target. Summed over the five distractors, this leads to an allocation of $60 \%$ of available resources to distractor processing, while the remaining $40 \%$ go into processing of the target. The blue encoding function in A1 shows the probability of target encoding in situation 2 .

Finally, situation 3 is the salient distractor condition, which makes target selection even more difficult. Now a salient distractor, uniquely colored and brighter than the target, is introduced. By virtue of its physical features, this distractor attracts significant attentional resources. Applying the simplified rate equation, we get 


$$
v=40 \times \frac{1}{(1 * 1)+(4 * .3)+(1 * 1.2)}=\frac{40}{3.4}=11.8
$$

The resources allocated to the salient distractor are $120 \%$ of those allocated to a target, and the target is only allotted less than $30 \%$ of all available resources. The yellow function in Fig. A1 shows the encoding function for situation 3.

The estimated model parameters in the current study (see Model fits) are obtained by minimizing residual sums of squares, using the Solver macro for Microsoft Excel. The models are fitted by varying three arousal-dependent free parameters: $s, w_{D}$ and $w_{S}$, for each value of the arousal manipulation, and a single arousal-independent parameter: $t_{0}$. The shape of the functions is constrained by the following equation:

$$
P=1-e^{-v(x, i) \times\left(t-t_{0}\right)}
$$

where $P$ is the probability of having encoded a target at time $t$. See Bundesen and Habekost (2008) for a detailed account of the assumptions and mathematics of TVA and Bundesen, Habekost and Kyllingsbæk (2011) for a neural interpretation of the theory. 


\section{Appendix B. Description of the IAPS pictures}

Table B1. Means and standard deviations of arousal and valence ratings of the IAPS pictures used in Experiments 1A \& 1B. The table shows descriptive statistics from the ratings collected by Lang et al. (2008).

\begin{tabular}{|l|l|c|c|c|c|c|c|}
\hline & & \multicolumn{2}{|c|}{ All } & \multicolumn{2}{c|}{ Female } & \multicolumn{2}{c|}{ Male } \\
\cline { 3 - 10 } Measure & Category & mean & SD & Mean & SD & mean & SD \\
\hline \multirow{2}{*}{ Arousal ratings } & neutral & 2.83 & .32 & 2.88 & .35 & 2.77 & .40 \\
\cline { 2 - 10 } & pleasant & 6.67 & .31 & 6.54 & .40 & 6.82 & .43 \\
\cline { 2 - 10 } & unpleasant & 6.75 & .30 & 7.01 & .39 & 6.44 & .32 \\
\hline \multirow{2}{*}{ Valence ratings } & neutral & 5.03 & .32 & 5.06 & .36 & 5.00 & .32 \\
& pleasant & 6.93 & .36 & 6.68 & .63 & 7.23 & .35 \\
\cline { 2 - 9 } & unpleasant & 1.95 & .38 & 1.63 & .35 & 2.35 & .46 \\
\hline
\end{tabular}

The arousing and neutral picture sets were tested for differences in luminance by estimating the perceived brightness of each pixel and then calculating the mean perceived brightness of each picture. Perceived brightness values were calculated using the coefficients of the rec.709 standard in the formula $L_{i}=.213 * R_{i}+.715 * G_{i}+.072 * B_{i}$, where $L_{i}$ is the luminance of a pixel $i$, and $R_{i}, G_{i}$ and $B_{i}$ represent the normalized input to each color channel for that pixel of the display. Weights of each RGB channel are set to simulate the perceived brightness. The difference between the mean perceived brightness of neutral (.436) and arousing (.404) pictures was not significant $(t(142)=1.30, p=.20)$, 
nor was there a difference in the picture-wise root-mean-square brightness contrast $(.255$

and .268, for neutral and arousing pictures, respectively; $t(142)=-1.24, p=.22)$.

Table B2. A list of all IAPS picture numbers used by category.

Neutral: 2036, 2102, 2104, 2190, 2221, 2393, 2397, 2411, 2513, 2840, 2850, 2870, 2880, 2890, 2980, 5120, 5130, 5471, 5510, 5530, 5534, 5731, 5740, 61507000, 7001, 7002, 7003, 7004, 7006, 7009, 7012, 7014, 7017, 7020, 7026, 7030, 7031, 7032, 7034, 7035, 7038, 7040, 7041, 7050, 7052, 7053, 70557056, 7059, 7060, 7080, 7090, 7100, 7110, 7140, 7150, 7160, 7161, 7179, 7185, 7205, 7217, 7233, 7300, 7490, 7491, 7500, 7547, 7705, 7950, 9210.

Pleasant: 4311, 4608, 4652, 4656, 4658, 4659, 4660, 4668, 4670, 4681, 4687, 4689, 4694, 4695, 4698, $4800,4810,5621,5626,5629,8030,8034,8158,8163,8178,8179,8180,8185,8186,8200,8206,8300$, $8370,8400,8490,8492$.

Unpleasant: $2683,2811,3000,3010,3030,3053,3059,3060,3068,3069,3071,3080,3110,3120,3130$, $3131,3150,3170,3212,3400,3500,3530,6313,6315,6560,8485,9050,9183,9187,9250,9410,9413$, $9414,9810,9910,9921$

\section{Appendix C. Supplementary Material}

The data associated with this article, as well as a brief presentation of subject-level model

fits, can be found in the online version [link to online version]

\section{References}

Allen, M., Frank, D., Schwarzkopf, D. S., Fardo, F., Winston, J. S., Hauser, T. U., \& Rees, G. (2016). Unexpected arousal modulates the influence of sensory noise on confidence. eLife, 5, 1-17. http://doi.org/10.7554/eLife.18103

Ásgeirsson, Á. G., Kristjánsson, Á., \& Bundesen, C. (2014). Independent priming of location and color in identification of briefly presented letters. Attention, Perception \& Psychophysics, 76, 40-8. http://doi.org/10.3758/s13414-013-0546-6

Ásgeirsson, Á. G., Kristjánsson, Á., \& Bundesen, C. (2015). Repetition priming in selective attention : A TVA analysis. Acta Psychologica, 160, 35-42. doi:10.1016/j.actpsy.2015.06.008

Arnsten, A. F. (2000). Stress impairs prefrontal cortical function in rats and monkeys: role of dopamine D1 and norepinephrine alpha-1 receptor mechanisms. Progress in Brain Research, 126, 183-192.

Bacon, S. J. (1974). Arousal and the range of cue utilization. Journal of Experimental Psychology, 102(1), 81-87.

Bogon, J., Finke, K., \& Stenneken, P. (2014). TVA-based assessment of visual attentional functions in developmental dyslexia. Frontiers in Psychology, 5(October), 1-6. 
http://doi.org/10.3389/fpsyg.2014.01172

Bradley, M. M., Miccoli, L., Escrig, M. A., \& Lang, P. J. (2008). The pupil as a measure of emotional arousal and autonomic activation. Psychophysiology, 45(4), 602-607. http://doi.org/10.1111/j.14698986.2008.00654.x

Brainard, D. H. (1997). The psychophysics toolbox. Spatial vision, 10, 433-436.

Brown, S. B. R. E., van Steenbergen, H., Band, G. P. H., de Rover, M., \& Nieuwenhuis, S. (2012). Functional significance of the emotion-related late positive potential. Frontiers in Human Neuroscience, 6, 1-12. http://doi.org/10.3389/fnhum.2012.00033

Bublak, P., Finke, K., Krummenacher, J., Preger, R., Kyllingsbaek, S., Müller, H. J., \& Schneider, W. X. (2005). Usability of a theory of visual attention (TVA) for parameter-based measurement of attention II: evidence from two patients with frontal or parietal damage. Journal of the International Neuropsychological Society: JINS, 11(7), 843-854. http://doi.org/10.1017/S1355617705050988

Bundesen, C. (1990). A theory of visual attention. Psychological Review, 97(4), 523-547. doi: $10.1037 / 0033-295 X .97 .4 .523$

Bundesen, C., \& Habekost, T. (2008). Principles of visual attention: Linking mind and brain. New York: Oxford University Press.

Bundesen, C., \& Habekost, T. (2014). Theory of visual attention (TVA). In A. C. Nobre, \& S. Kastner (Eds.), The Oxford handbook of attention. New York, NY: Oxford University Press.

Bundesen, C., Habekost, T., \& Kyllingsbæk, S. (2011). A neural theory of visual attention and short-term memory (NTVA). Neuropsychologia, 49(6), 1446-1457. doi:10.1016/j.neuropsychologia.2010.12.006

Bundesen, C., Vangkilde, S., \& Habekost, T. (2015). Components of visual bias: a multiplicative hypothesis. Annals of the New York Academy of Sciences, 1339(1), 116-124. doi:10.1111/nyas.12665

Calderon, D. P., Kilinc, M., Maritan, A., Banavar, J. R., \& Pfaff, D. (2016). Generalized CNS arousal: An elementary force within the vertebrate nervous system. Neuroscience and Biobehavioral Reviews, 68, 167-176. doi: 10.1016/j.neubiorev.2016.05.014.

Cavanagh, J. F., Wiecki, T. V, Kochar, A., \& Frank, M. J. (2014). Eye tracking and pupillometry are indicators of dissociable latent decision processes. Journal of Experimental Psychology. General, 143(4), 1476-88. http://doi.org/10.1037/a0035813

Clewett, D., Sakaki, M., Nielsen, S., Petzinger, G., \& Mather, M. (2017). Noradrenergic mechanisms of arousal's bidirectional effects on episodic memory. Neurobiology of Learning and Memory, 137, 1-14. http://doi.org/10.1016/j.nlm.2016.10.017

Codispoti, M., Ferrari, V., \& Bradley, M. M. (2006). Repetitive picture processing: Autonomic and cortical correlates. Brain Research, 1068(1), 213-220. doi:10.1016/j.brainres.2005.11.009

Cuthbert, B. N., Schupp, H. T., Bradley, M. M., Birbaumer, N., \& Lang, P. J. (2000). Brain potentials in affective picture processing: Covariation with autonomic arousal and affective report. Biological Psychology, 52(2), 95-111. http://doi.org/10.1016/S0301-0511(99)00044-7

Delorme, A., \& Makeig, S. (2004). EEGLAB: an open source toolbox for analysis of single-trail EEG dynamics including independent component analysis. Journal of Neuroscience Methods, 134, 9-21. http://doi.org/10.1016/j.jneumeth.2003.10.009

Desimone, R., \& Duncan, J. S. (1995). Neural mechanisms of selective visual attention. Annual Review of Neuroscience, 18, 193-222. http://doi.org/10.1146/annurev.ne.18.030195.001205

Duncan, J., Bundesen, C., Olson, a, Humphreys, G., Chavda, S., \& Shibuya, H. (1999). Systematic analysis of deficits in visual attention. Journal of Experimental Psychology. General, 128(4), 450-478. http://doi.org/10.1037/0096-3445.128.4.450

Easterbrook, J. A. (1959). The effect of emotion on cue utilization and the organization of behavior. Psychological Review, 66(3), 183-201. http://doi.org/10.1037/h0047707

Eldar, E., Cohen, J. D., \& Niv, Y. (2013). The effects of neural gain on attention and learning. Nature Neuroscience, 16(June), 1146-53. http://doi.org/10.1038/nn.3428

Eysenck, M. W. (1976). Arousal, Learning, and Memory. Psychological Bulletin, 83(3), 389-404. http://doi.org/10.1037/0033-2909.83.3.389

Fecteau, J. H., \& Munoz, D. P. (2006). Salience, relevance, and firing: a priority map for target selection. Trends in Cognitive Sciences, 10, 382-390. 
Gerdes, A. B. M., Wieser, M. J., Bublatzky, F., Kusay, A., Plichta, M. M., \& Alpers, G. W. (2013). Emotional sounds modulate early neural processing of emotional pictures. Frontiers in Psychology, 4(October), 741. http://doi.org/10.3389/fpsyg.2013.00741

Habekost, T. (2015). Clinical TVA-based studies: A general review. Frontiers in Psychology, 6(MAR), 118. http://doi.org/10.3389/fpsyg.2015.00290

Hackley, S. a., \& Valle-Inclán, F. (2003). Which stages of processing are speeded by a warning signal? Biological Psychology, 64, 27-45. http://doi.org/10.1016/S0301-0511(03)00101-7

Hajcak, G., MacNamara, A., \& Olvet, D. M. (2010). Event-related potentials, emotion, and emotion regulation: an integrative review. Developmental neuropsychology, 35(2), 129-155.

Hanoch, Y., \& Vitouch, O. (2004). When less is more: information, emotional arousal and the ecological reframing of the Yerkes-Dodson law. Theory \& Psychology, 14(4), 427-452. http://doi.org/10.1177/0959354304044918

Hockey, G. R. J. (1970). Signal probability and spatial location as possible bases for increased selectivity in noise. Quarterly Journal of Experimental Psychology, 22(March 2015), 37-42. http://doi.org/10.1080/14640747008401899

Hockey G. R. J., \& Hamilton, P. (1970). Arousal and information selection in short-term memory. Nature, 226, 866-867. doi: $10.1038 / 226866 \mathrm{a} 0$

Jarosz, A. F., \& Wiley, J. (2014). What are the odds? A practical guide to computing and reporting Bayes Factors. The Journal of Problem Solving, 7, 2-9. http://doi.org/10.7771/1932-6246.1167

JASP Team (2016). JASP (Version 0.7.5.5)[Computer software].

Jeffreys, H. (1961). The theory of probability. Oxford: Oxford University Press.

Jepma, M., Wagenmakers, E.-J., Band, G. P. H., \& Nieuwenhuis, S. (2009). The effects of accessory stimuli on information processing: evidence from electrophysiology and a diffusion model analysis. Journal of Cognitive Neuroscience, 21(5), 847-864. http://doi.org/10.1162/jocn.2009.21063

Joshi, S., Li, Y., Kalwani, R. M., \& Gold, J. I. (2016). Relationships between Pupil Diameter and Neuronal Activity in the Locus Coeruleus, Colliculi, and Cingulate Cortex. Neuron, 89(1), 221-234. http://doi.org/10.1016/j.neuron.2015.11.028

Kahneman, D. (1973). Attention and effort (p. 246). Englewood Cliffs, NJ: Prentice-Hall.

Kleiner, M., Brainard, D., Pelli, D., Ingling, A., Murray, R., \& Broussard, C. (2007). What's new in Psychtoolbox-3. Perception, 36(14), 1.

Knapen, T., de Gee, J. W., Brascamp, J., Nuiten, S., Hoppenbrouwers, S., \& Theeuwes, J. (2016). Cognitive and ocular factors jointly determine pupil responses under equiluminance. Plos One, 11(5), e0155574. http://doi.org/10.1371/journal.pone.0155574

Koster, E. H., Crombez, G., Verschuere, B., \& De Houwer, J. (2004). Selective attention to threat in the dot probe paradigm: Differentiating vigilance and difficulty to disengage. Behaviour research and therapy, 42(10), 1183-1192.

Kristjánsson, Á., Óladóttir, B., \& Most, S. B. (2012). "Hot” facilitation of "cool” processing: Emotional distraction can enhance priming of visual search. Journal of Experimental Psychology: Human Perception and Performance. 39(1), 298-306. doi:10.1037/a0028683

Kyllingsbæk, S., \& Bundesen, C. (2009). Changing change detection: improving the reliability of measures of visual short-term memory capacity. Psychonomic Bulletin \& Review, 16(6), 1000-1010. doi:10.3758/PBR.16.6.1000

Lang, P.J., Bradley, M.M., \& Cuthbert, B.N. (2008). International affective picture system (IAPS): Affective ratings of pictures and instruction manual. Technical Report A-8. University of Florida, Gainesville, FL.

Lawrence, M. A, \& Klein, R. M. (2013). Isolating exogenous and endogenous modes of temporal attention. J Exp Psychol Gen, 142(2), 560-572. http://doi.org/10.1037/a0029023

Lee, T. H., Baek, J., Lu, Z. L., \& Mather, M. (2014). How arousal modulates the visual contrast sensitivity function. Emotion, 14(5), 978-984. http://doi.org/10.1037/a0037047

Lee, T. H., Itti, L., \& Mather, M. (2012). Evidence for arousal-biased competition in perceptual learning. Frontiers in Psychology, 3, 1-9. http://doi.org/10.3389/fpsyg.2012.00241

Mather, M. (2007). Emotional arousal and memory binding: An object-based framework. Perspectives on 
Psychological Science, 2(1), 33-52. http://doi.org/10.1111/j.1745-6916.2007.00028.x

Mather, M., Clewett, D., Sakaki, M., \& Harley, C. W. (2016). Norepinephrine ignites local hot spots of neuronal excitation: How arousal amplifies selectivity in perception and memory. Behavioral Brain Science, 39: e200.

Mather, M., \& Sutherland, M. R. (2011). Arousal-Biased Competition in Perception and Memory. Perspectives on Psychological Science, 6, 114-133. doi:10.1177/1745691611400234

Matthias, E., Bublak, P., Müller, H. J., Schneider, W. X., Krummenacher, J., \& Finke, K. (2010). The influence of alertness on spatial and nonspatial components of visual attention. Journal of Experimental Psychology. Human Perception and Performance, 36(1), 38-56. http://doi.org/10.1037/a0017602

Matzke, D., Nieuwenhuis, S., van Rijn, H., Slagter, H. a, van der Molen, M. W., \& Wagenmakers, E.-J. (2015). The effect of horizontal eye movements on free recall: A preregistered adversarial collaboration. Journal of Experimental Psychology: General, 144(1), e1-e15. http://doi.org/10.1037/xge0000038

Murphy, P. R., Boonstra, E., \& Nieuwenhuis, S. (2016). Time-dependent urgency via global gain modulation during human perceptual choice. Nature Communications, 7(May), 1-14. http://doi.org/10.1038/ncomms 13526

Murphy, P. R., O’Connell, R. G., O’Sullivan, M., Robertson, I. H., \& Balsters, J. H. (2014). Pupil diameter covaries with BOLD activity in human locus coeruleus. Human Brain Mapping, 35(8), 4140-4154. http://doi.org/10.1002/hbm.22466

Murphy, P. R., Vandekerckhove, J., \& Nieuwenhuis, S. (2014). Pupil-Linked Arousal Determines Variability in Perceptual Decision Making. PLoS Computational Biology, 10(9), e1003854. http://doi.org/10.1371/journal.pcbi.1003854

Nassar, M. R., Rumsey, K. M., Wilson, R. C., Parikh, K., Heasly, B., \& Gold, J. I. (2012). Rational regulation of learning dynamics by pupil-linked arousal systems. Nature Neuroscience, 15(7), 1040 1046. http://doi.org/10.1038/nn.3130

Nieuwenhuis, S., de Geus, E.J., \& Aston-Jones, G. (2011). The anatomical and functional relationship between the P3 and autonomic components of the orienting response. Psychophysiology, 48, 162-175.

Nordfang, M., Dyrholm, M., \& Bundesen, C. (2013). Identifying bottom-up and top-down components of attentional weight by experimental analysis and computational modeling. Journal of Experimental Psychology: General, 142(2), 510-535. http://doi.org/10.1037/a0029631

Pelli, D. G. (1997). The VideoToolbox software for visual psychophysics: Transforming numbers into movies. Spatial vision, 10(4), 437-442.

Petersen, A., Petersen, A. H., Bundesen, C., Vangkilde, S., \& Habekost, T. (2017). The effect of phasic auditory alerting on visual perception. Cognition, 165, 73-81. https://doi.org/10.1016/j.cognition.2017.04.004

Poth, C. H., Petersen, A., Bundesen, C., \& Schneider, W. X. (2014). Effects of monitoring for visual events on distinct components of attention. Frontiers in Psychology, 5(September), 1-14. https://doi.org/10.3389/fpsyg.2014.00930

Robbins, T. W. (1997). Arousal systems and attentional processes. Biological psychology, 45(1), 57-71.

Rouder, J. N., Morey, R. D., Verhagen, J., Swagman, A. R., \& Wagenmakers, E.-J. (in press). Bayesian Analysis of Factorial Designs. Psychological Methods. http://dx.doi.org/10.1037/met0000057

Sakaki, M., Fryer, K., \& Mather, M. (2014). Emotion strengthens high-priority memory traces but weakens low-priority memory traces. Psychological Science, 25, 387-395. http://doi.org/10.1177/0956797613504784

Servan-Schreiber, D., Printz, H., \& Cohen, J. D. (1990). A network model of catecholamine effects: gain, signal-to-noise ratio, and behavior. Science, 249(4971), 892-895. http://doi.org/10.1126/science.2392679

Shibuya, H., \& Bundesen, C. (1988). Visual selection from multielement displays: Measuring and modeling effects of exposure duration. Journal of Experimental Psychology: Human Perception and Performance, 14(4), 591-600. http://doi.org/10.1037/0096-1523.14.4.591

Sørensen, T. A., Vangkilde, S., \& Bundesen, C. (2015). Components of attention modulated by temporal 
expectation. Journal of Experimental Psychology: Learning, Memory, and Cognition, 41(1), 178-192. http://doi.org/10.1037/a0037268

Sutherland, M. R., \& Mather, M. (2012). Negative arousal amplifies the effects of saliency in short-term memory. Emotion, 12(6), 1367-1372. doi:10.1037/a0027860

Sutherland, M. R., McQuiggan, D. A., Ryan, J. D., \& Mather, M. (2017). Perceptual salience does not influence emotional arousal's impairing effects on top-down attention. Emotion, 17, 700-706. doi: $10.1037 / \mathrm{emo} 0000245$

Tona, K.-D., Murphy, Peter. R., Brown, S. B.R.E., \& Nieuwenhuis, S. (2016), The accessory stimulus effect is mediated by phasic arousal: A pupillometry study. Psychophysiol, 53, 1108-1113. doi:10.1111/psyp.12653

van den Brink, R.L., Murphy, P.R., \& Nieuwenhuis, S. (2016). Pupil diameter tracks lapses of attention. PLOS One, 11(10): e 0165274. doi:10.1371/journal.pone.0165274

Vangkilde, S., Coull, J. T., \& Bundesen, C. (2012). Great expectations: Temporal expectation modulates perceptual processing speed. Journal of Experimental Psychology: Human Perception and Performance, 38(5), 1183-1191. http://doi.org/10.1037/a0026343

Vangkilde, S., Petersen, A., \& Bundesen, C. (2013). Temporal expectancy in the context of a theory of visual attention. Philosophical Transactions of the Royal Society B: Biological Sciences, 368(1628), 20130054-20130054. http://doi.org/10.1098/rstb.2013.0054

Vinck, M., Batista-Brito, R., Knoblich, U., \& Cardin, J. a. (2015). Arousal and locomotion make distinct contributions to cortical activity patterns and visual encoding. Neuron, 86(3), 740-754. http://doi.org/10.1016/j.neuron.2015.03.028

Warren, C. M., Eldar, E., van den Brink, R. L., Tona, K.-D., van der Wee, N. J., Giltay, E. J., van Noorden, M. S., Bosch, J. A., Wilson, R. C., Cohen, J. D., \& Nieuwenhuis, S. (2016). Catecholamine-mediated increases in gain enhance the precision of cortical representations. Journal of Neuroscience, 36(21), 5699-5708. http://doi.org/10.1523/JNEUROSCI.3475-15.2016

Warren, C.M., Murphy, P.R., \& Nieuwenhuis, S. (2016). Cognitive control, dynamic salience and the imperative toward computational accounts of neuromodulatory function. Behavioral and Brain Sciences, 39, e227.

Wichary, S., Mata, R., \& Rieskamp, J. (2015). Probabilistic inferences under emotional stress: How arousal affects decision processes. Journal of Behavioral Decision Making, 538(August 2015), n/a-n/a. http://doi.org/10.1002/bdm.1896

Wiegand, I., Petersen, A., Lasner, J., Finke, K, \& Habekost, T. (2016). Auditory alerting enhances visual attentional processing: Evidence from computational modeling and event-related lateralizations. Journal of Vision, 16, 615. doi:10.1167/16.12.615

Wiegand, I., Töllner, T., Dyrholm, M., Müller, H. J., Bundesen, C., \& Finke, K. (2014). Neural correlates of age-related decline and compensation in visual attention capacity. Neurobiology of Aging, 35(9), 2161-2173. http://doi.org/10.1016/j.neurobiolaging.2014.02.023 


\section{Figure Legends}

Fig. 1. The general progression of a trial in all three experiments. The left panel shows stages of the procedure of all experiments. Stages 3-5 show the different conditions of distraction: the target alone condition (3), the homogeneous distractor condition (4), and the salient distractor condition (5). Note that the $T$ 's, $D$ 's and $S$ are symbolic of targets, distractors and salient distractor. In the experiment, the identities of letter stimuli were randomly chosen from all letters of the English alphabet. The right panel shows the arousal manipulations that were unique to each experiment. In Experiments 1A, 1B (arousing pictures) and 2 (an alerting tone), the arousing - or control - stimuli were inserted at stage 2. In Experiments $3 \mathrm{~A}$ and $3 \mathrm{~B}$, white noise was present/absent throughout whole blocks of the experimental task. Stimuli are not drawn to scale nor in their true colors (see Stimuli and Apparatus for details).

Fig. 2. All IAPS pictures plotted by arousal rating and valence rating from Lang et al. (2008). The three colored clusters represent the pictures in each of the three categories used in the current study.

Fig. 3. The primary results from Experiments $1 \mathrm{~A}, 2$ and $3 \mathrm{~A}$. Error bars show \pm 1 SEM. Note that betweenexperiment comparisons are complicated by minor differences in experimental parameters, such as duration of pre-stimulus fixation and exposure durations. See the Method sections of each experiment for details.

Fig. 4. Grand-average ERPs from the six participants in Experiment 1B. The large and protracted positivity following arousing pictures is clear in short (solid lines), and long (dotted lines) ISI trials, and remains significant in the windows of interest, immediately before target presentation. $*: p<.05$.

Fig. 5. Results from Experiment 3B. Data points show the mean pupil diameters ( $z$-scored within-subject) after controlling for time-on-task effects.

Fig. 6. A-C: Empirical (points) and simulated performance (lines) of a 7-parameter TVA model, which was fitted to the data collapsed across all participants. Distraction levels are abbreviated as TA: target alone, HD: homogeneous distractors, and SD: salient distractor. D: The relative allocation of processing resources for each stimulus type, as estimated by the TVA model. Note that the blue area of the bars represents 4 blue distractors in the SD condition, but 5 blue distractors in the HD condition. 


\section{Tables}

Table 1. TVA parameter estimates from a 7-parameter model of the data from Experiments 1A, 2 and 3A.

\begin{tabular}{|c|c|c|c|c|c|c|}
\hline \multirow[b]{2}{*}{ Parameter } & \multicolumn{2}{|c|}{ IAPS } & \multicolumn{2}{|c|}{ Alerting Tone } & \multicolumn{2}{|c|}{ White Noise } \\
\hline & Neutral & Arousing & Off & On & Off & On \\
\hline Processing speed $(s)$ & 59.4 & 59.8 & 46.4 & 69.3 & 48.0 & 46.3 \\
\hline Weight of blue distractor (wblue) & .235 & .235 & .304 & .381 & .300 & .289 \\
\hline Weight of yellow distractor ( $w_{\text {yellow }}$ ) & .866 & .814 & 1.352 & 1.119 & .844 & .716 \\
\hline Threshold for visual perception $\left(t_{0}\right)$ & \multicolumn{2}{|c|}{23.8} & \multicolumn{2}{|c|}{25.0} & \multicolumn{2}{|c|}{25.7} \\
\hline$R^{2}$ & \multicolumn{2}{|c|}{.986} & \multicolumn{2}{|c|}{.994} & \multicolumn{2}{|c|}{.993} \\
\hline
\end{tabular}

Table B1. Means and standard deviations of arousal and valence ratings of the IAPS pictures used in Experiments $1 \mathrm{~A} \& 1 \mathrm{~B}$. The table shows descriptive statistics from the ratings collected by Lang et al. (2008).

\begin{tabular}{|c|c|c|c|c|c|c|c|}
\hline \multirow[b]{2}{*}{ Measure } & \multirow[b]{2}{*}{ Category } & \multicolumn{2}{|c|}{ All } & \multicolumn{2}{|c|}{ Female } & \multicolumn{2}{|c|}{ Male } \\
\hline & & mean & SD & Mean & SD & mean & $\mathrm{SD}$ \\
\hline \multirow{3}{*}{ Arousal ratings } & neutral & 2.83 & .32 & 2.88 & .35 & 2.77 & .40 \\
\hline & pleasant & 6.67 & .31 & 6.54 & .40 & 6.82 & .43 \\
\hline & unpleasant & 6.75 & .30 & 7.01 & .39 & 6.44 & .32 \\
\hline \multirow{3}{*}{ Valence ratings } & neutral & 5.03 & .32 & 5.06 & .36 & 5.00 & .32 \\
\hline & pleasant & 6.93 & .36 & 6.68 & .63 & 7.23 & .35 \\
\hline & unpleasant & 1.95 & .38 & 1.63 & .35 & 2.35 & .46 \\
\hline
\end{tabular}

